\title{
The Life Cycle of Northern Hemisphere Downward Wave Coupling between the Stratosphere and Troposphere
}

\author{
TIFFANY A. SHAW \\ Department of Earth and Environmental Sciences, Lamont-Doherty Earth Observatory, Palisades, and Department of \\ Applied Physics and Applied Mathematics, Columbia University, New York, New York \\ JUDITH PERLWITZ* \\ Cooperative Institute for Research in Environmental Sciences, University of Colorado, Boulder, Colorado
}

(Manuscript received 9 May 2012, in final form 31 August 2012)

\begin{abstract}
The life cycle of Northern Hemisphere downward wave coupling between the stratosphere and troposphere via wave reflection is analyzed. Downward wave coupling events are defined by extreme negative values of a wave coupling index based on the leading principal component of the daily wave- 1 heat flux at $30 \mathrm{hPa}$. The life cycle occurs over a 28-day period. In the stratosphere there is a transition from positive to negative total wave-1 heat flux and westward to eastward phase tilt with height of the wave-1 geopotential height field. In addition, the zonal-mean zonal wind in the upper stratosphere weakens leading to negative vertical shear.

Following the evolution in the stratosphere there is a shift toward the positive phase of the North Atlantic Oscillation (NAO) in the troposphere. The pattern develops from a large westward-propagating wave-1 anomaly in the high-latitude North Atlantic sector. The subsequent equatorward propagation leads to a positive anomaly in midlatitudes. The near-surface temperature and circulation anomalies are consistent with a positive NAO phase. The results suggest that wave reflection events can directly influence tropospheric weather.

Finally, winter seasons dominated by extreme wave coupling and stratospheric vortex events are compared. The largest impacts in the troposphere occur during the extreme negative seasons for both indices, namely seasons with multiple wave reflection events leading to a positive NAO phase or seasons with major sudden stratospheric warmings (weak vortex) leading to a negative NAO phase. The results reveal that the dynamical coupling between the stratosphere and NAO involves distinct dynamical mechanisms that can only be characterized by separate wave coupling and vortex indices.
\end{abstract}

\section{Introduction}

Dynamical coupling between the stratosphere and troposphere is a key component of atmospheric variability in the winter hemisphere. Understanding the mechanisms involved in this coupling and its impact on tropospheric weather and climate is an important

\footnotetext{
* Additional affiliation: NOAA/Earth System Research Laboratory, Physical Sciences Division, Boulder, Colorado.

Corresponding author address: Dr. Tiffany A. Shaw, Department of Earth and Environmental Sciences and Department of Applied Physics and Applied Mathematics, Columbia University, P.O. Box 1000, 61 Route 9W, Palisades, NY 10964.

E-mail: tas2163@columbia.edu
}

topic of current research (Shaw and Shepherd 2008; Gerber et al. 2012). It is well known that stratospheretroposphere coupling is driven by the upward propagation of planetary-scale waves generated in the troposphere. A significant amount of research has been focused on understanding the coupling during weak stratospheric vortex events, for example, sudden stratospheric warming events, that involve the absorption of wave activity in the stratosphere and the downward migration of zonal-mean zonal wind and temperature anomalies (e.g., Baldwin and Dunkerton 1999, 2001; Christiansen 2001; Plumb and Semeniuk 2003). When the zonalmean anomalies reach the lower stratosphere/upper troposphere they can initiate baroclinic eddy responses that subsequently produce anomalies in the tropospheric circulation, for example, meridional shifts of the jet, that 
can be maintained by eddy feedbacks (e.g., Polvani and Kushner 2002; Song and Robinson 2004; Limpasuvan et al. 2004) and produce surface temperature and mean sea level pressure anomalies. In addition, weak vortex events produce potential vorticity anomalies in the stratosphere that can directly impact the troposphere via hydrostatic and geostrophic adjustment (Hartley et al. 1998; Black 2002; Ambaum and Hoskins 2002). Baldwin and Dunkerton (2001) showed that strong stratospheric vortex events could also impact the troposphere. Polar vortex intensification is accompanied by equatorward propagation of wave activity in the stratosphere (Hartmann et al. 2000) with the impacts in the troposphere resulting from the subsequent hydrostatic and geostrophic adjustment of the vortex (Ambaum and Hoskins 2002).

In addition to coupling involving extreme vortex events, Perlwitz and Harnik $(2003,2004)$ and Shaw et al. (2010 hereafter SPH10) showed that wave reflection in the stratosphere can impact the tropospheric wave structure, a process called "downward wave coupling." SPH10 used the statistical cross-correlation technique employed by Randel (1987) to show that upward wave-1 coupling from 500 to $30 \mathrm{hPa}$ occurs over a 5-day period and is followed by downward wave- 1 coupling from 30 to $500 \mathrm{hPa}$ over a 5-day period. In the Northern Hemisphere, downward wave coupling maximized during January-March (JFM). Note that the wave coupling time scales are much shorter than time scales associated with the downward migration of zonal-mean anomalies during extreme vortex events. SPH10 showed that downward wave- 1 coupling is associated with a particular configuration of the stratospheric basic state that is favorable for wave- 1 reflection in the stratosphere. The basic-state configuration involves a meridional wave evanescence region in the subtropical stratosphere and a vertical wave evanescence region in the upper stratosphere (SPH10). The vertical wave evanescence coincides with a region of negative vertical zonal wind shear (Perlwitz and Harnik 2003, 2004) whereas the meridional wave evanescence region is associated with negative meridional zonal wind shear. The configuration channels wave activity upward from the troposphere to the stratosphere and, upon wave reflection, from the stratosphere to the troposphere. Perlwitz and Harnik (2004) showed that individual winters in the Northern Hemisphere could be characterized as being dominated by wave reflection or wave absorptiontype stratosphere-troposphere coupling, each type being associated with distinct stratospheric basic states.

All stratosphere-troposphere coupling events, whether they involve anomalous vortex states or downward wave coupling, originate as events of upward wave propagation (upward wave coupling) events from the troposphere (Haynes 2005). Polvani and Waugh (2004) and
Limpasuvan et al. (2004) showed that weak vortex events were preceded by anomalous positive 40 -day, $45^{\circ}-75^{\circ} \mathrm{N}$ averaged $100-\mathrm{hPa}$ (meridional) heat flux events that were extreme (far from the mean). They argued that weak vortex events are "true events" because they were associated with extreme positive heat flux events, that is, enhanced propagation of planetary wave activity into the stratosphere. In contrast, the relationship between strong vortex and heat flux events is less clear. Polvani and Waugh showed that strong vortex events were associated with anomalous weakly negative 40 -day, $45^{\circ}-$ $75^{\circ} \mathrm{N}$-averaged $100-\mathrm{hPa}$ heat flux (see their Fig. 1). However, an anomalous negative heat flux event is not associated with a distinct dynamical mechanism since it can imply either weakened upward wave coupling or downward wave coupling (i.e., wave reflection). The latter occurs if the total (climatology plus anomaly) heat flux is negative because the total heat flux is proportional to the vertical group velocity. Recall that the meridional heat flux contributes to the vertical EliassenPalm flux, which is equal to the vertical group velocity times the wave-activity density in the quasigeostrophic and small-amplitude limits. The relationship between negative total heat flux events and strong vortex events has not been investigated previously. A complete characterization of stratosphere-troposphere coupling requires a better understanding of negative heat flux events, their relationship to vortex events, and their impact on the troposphere.

Here we use the daily wave- 1 heat flux to isolate and analyze downward wave coupling events during JFM in the Northern Hemisphere. The events are defined by extreme negative values of a daily wave coupling index equal to the standardized principal component (PC) of the leading empirical orthogonal function (EOF) of the wave-1 heat flux. The PC time series is utilized instead of the daily heat flux anomaly averaged over a specified latitudinal band from $45^{\circ}$ to $75^{\circ} \mathrm{N}$, as in Newman et al. (2001) and Polvani and Waugh (2004), because it encodes the spatial variability via the EOF and the temporal variability via the PC. Note also that we use a daily index instead of a long time-scale-averaged index as in Polvani and Waugh because upward and downward wave coupling involves short time scales.

Section 2 discusses the data and analysis methods. Section 3 discusses the composite life cycle of downward wave coupling events in the stratosphere and their impact on tropospheric weather. In addition, the seasonal impacts of downward wave coupling on the troposphere and the relationship to the conventional weak/strong vortex paradigms of stratosphere-troposphere coupling are analyzed. The results are summarized and discussed in section 4 . 


\section{Data and event definition}

The data used in this study are the daily threedimensional zonal and meridional wind, temperature, and geopotential height from the European Centre for Medium-Range Weather Forecasts Interim (ERAInterim) dataset from 1979 to 2011 (Dee et al. 2011). The data are provided on 37 pressure levels with a horizontal resolution of $1.5^{\circ}$.

The focus of the analysis is on downward wave coupling associated with planetary waves with zonal wavenumber 1 (downward wave-1 coupling). SPH10 showed that downward wave-1 coupling maximizes during JFM. Downward wave-2 coupling also occurs in the Northern Hemisphere but it is less frequent (SPH10) and consequently it is more difficult to quantify its impact on the troposphere based on a record as short as the ERAInterim dataset.

The life cycle analysis is based on the principal component of the leading EOF of the zonal-mean wave-1 heat flux at $30 \mathrm{hPa}$ in the Northern Hemisphere. The $30-\mathrm{hPa}$ level is chosen so that the cause of downward wave coupling events can be clearly attributed to events originating in the stratosphere. The leading EOF is calculated using the entire 12 045-day time series (365 days and 33 years) from $20^{\circ}$ to $90^{\circ} \mathrm{N}$ with a meridional weighting following Baldwin et al. (2009). The leading EOF explains $84 \%$ of the total variance and is well separated from higher modes according to the criterion of North et al. (1982). The magnitude of the PC, which is defined over the whole year, is largest during winter, consistent with vertical planetary wave propagation into the stratosphere during this season. As a result, the standard deviation of the $\mathrm{PC}$ during $\mathrm{JFM}$ is $\sigma_{\mathrm{JFM}}=1.7$, which is larger than the standard deviation for all days (e.g., $\sigma=$ 1.0; as per the definition from the EOF analysis).

The climatological JFM wave-1 heat flux is positive from the surface to the upper stratosphere (Fig. 1, topleft panel) and suggests that the climatology is dominated by upward wave propagation, which is consistent with SPH10 who showed that downward wave coupling occurs as part of the intraseasonal variability. Figure 1 (top-middle panel) shows the leading spatial pattern of variability of wave-1 heat flux determined by regressing the wave- 1 heat flux anomalies at all levels on the standardized 30-hPa PC time series. This heat flux pattern is shifted toward high latitudes relative to the climatology and exhibits a dipole pattern in the vertical. It is positive in the stratosphere and negative in the troposphere. The goal of this study is isolate downward coupling events (i.e., time periods when the total heat flux in high latitudes is negative indicating a downward group velocity). A PC value equal to $-\sigma_{\mathrm{JFM}}$ is sufficient to produce a total negative heat flux, for example, the sum of the climatological heat flux pattern and $-\sigma_{\mathrm{JFM}}$ times the regression pattern is negative in high latitudes (Fig. 1, top-right panel). Thus, an extreme PC value of $-\sigma_{\mathrm{JFM}}$ can be used as a threshold for downward wave coupling events. The histogram of JFM PC values (Fig. 1, bottom panel) illustrates that downward wave coupling events are fairly common: the number of JFM days from 1979 to 2011 with a PC value $<-\sigma_{\mathrm{JFM}}\left(>+\sigma_{\mathrm{JFM}}\right)$ is 332 (418).

To create a composite life cycle of downward wave coupling we define events based on a wave coupling index equal to the 5-day smoothed leading PC time series of daily heat flux anomalies at $30 \mathrm{hPa}$. The smoothing allows for a clear assessment of the central dates of the events. An individual downward wave coupling event is identified during a given JFM season when the minimum value of the wave coupling index during the season is less than or equal to $-1.5 \sigma_{\mathrm{JFM}}$. Note that this threshold ensures that the event represents an extreme value of the PC during JFM and a negative total heat flux in high latitudes at $30 \mathrm{hPa}$ (see Fig. 1, top-right panel). In addition, the event must last at least 5 days, for example, the index must be less than $\sigma_{\mathrm{JFM}}$ for 2 days before and after the event, and the index value of the wave- 1 heat flux anomaly time series projected onto the regression pattern at $100 \mathrm{hPa}$ must be less than $\sigma_{\mathrm{JFM}}$ sometime 5 days following the event. The central date of the event (day 0) is defined by the day that the index crosses -1.0. Events defined using the PC at 50 and $100 \mathrm{hPa}$ exhibit similar qualitative behavior as those defined at $30 \mathrm{hPa}$.

\section{Results}

According to the criteria of section 2, 14 downward wave coupling events occurred during the 33 JFM seasons from 1979 to 2011. The central dates of the individual events are listed in Table 1 along with the minimum total wave- 1 heat flux from $40^{\circ}$ to $90^{\circ} \mathrm{N}$ during the event. The life cycle of the composite event is divided into four 7-day stages; stage 1 ( -12 to -6 days), stage 2 ( -5 to +1 days), stage 3 ( 2 to 8 days), and stage 4 (9 to 15 days). A 5-day smoothing is applied to all data. The life cycle approach is similar to Limpasuvan et al. (2004) who studied the life cycle of Northern Hemisphere sudden stratospheric warming events; however, the time scale of the stages is much shorter here [7 days vs 15 days in Limpasuvan et al. (2004)]. Note that the sudden warming events were defined using a stratospheric zonal index based on the 15-day low-pass filtered leading PC time series of daily zonal-mean zonal wind anomalies at $50 \mathrm{hPa}$. 

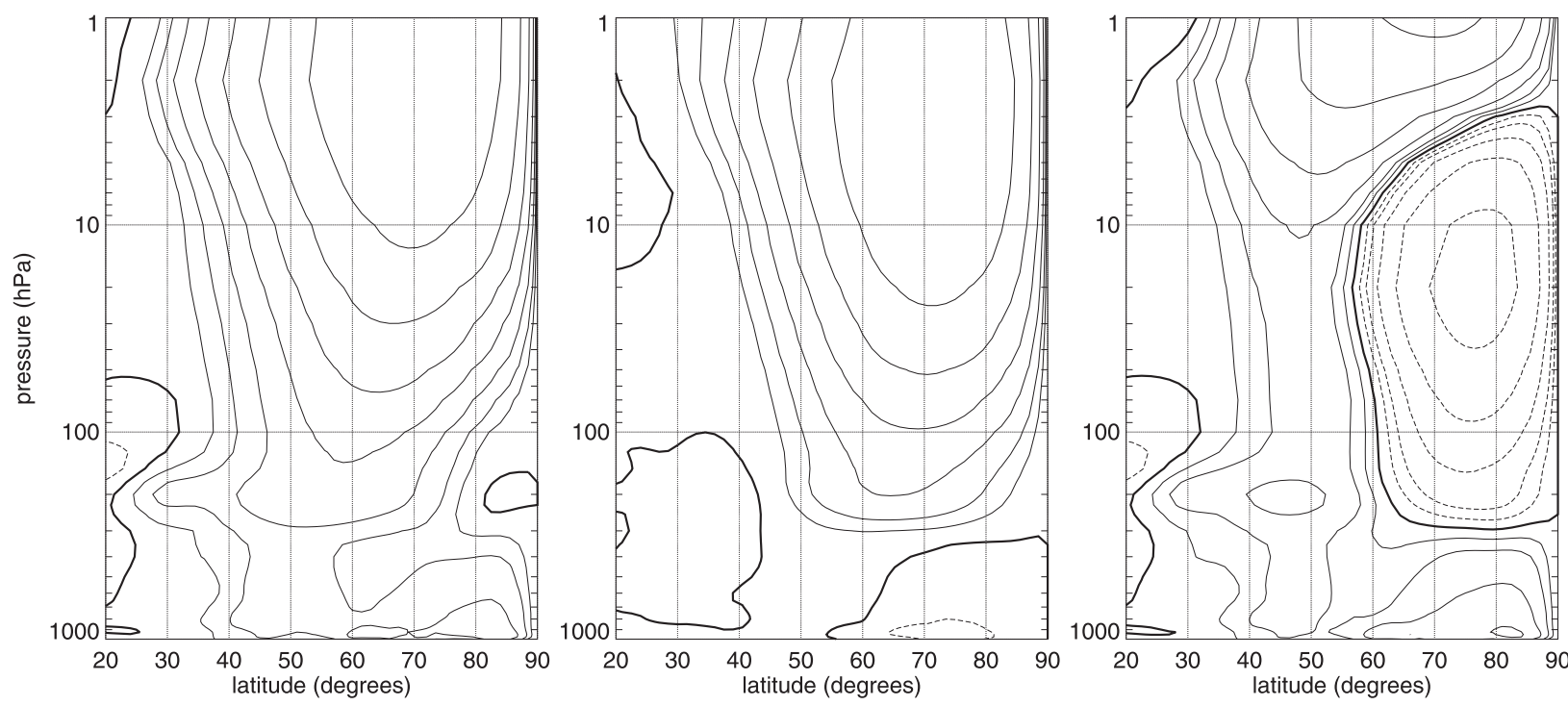

pc 30hPa JFM

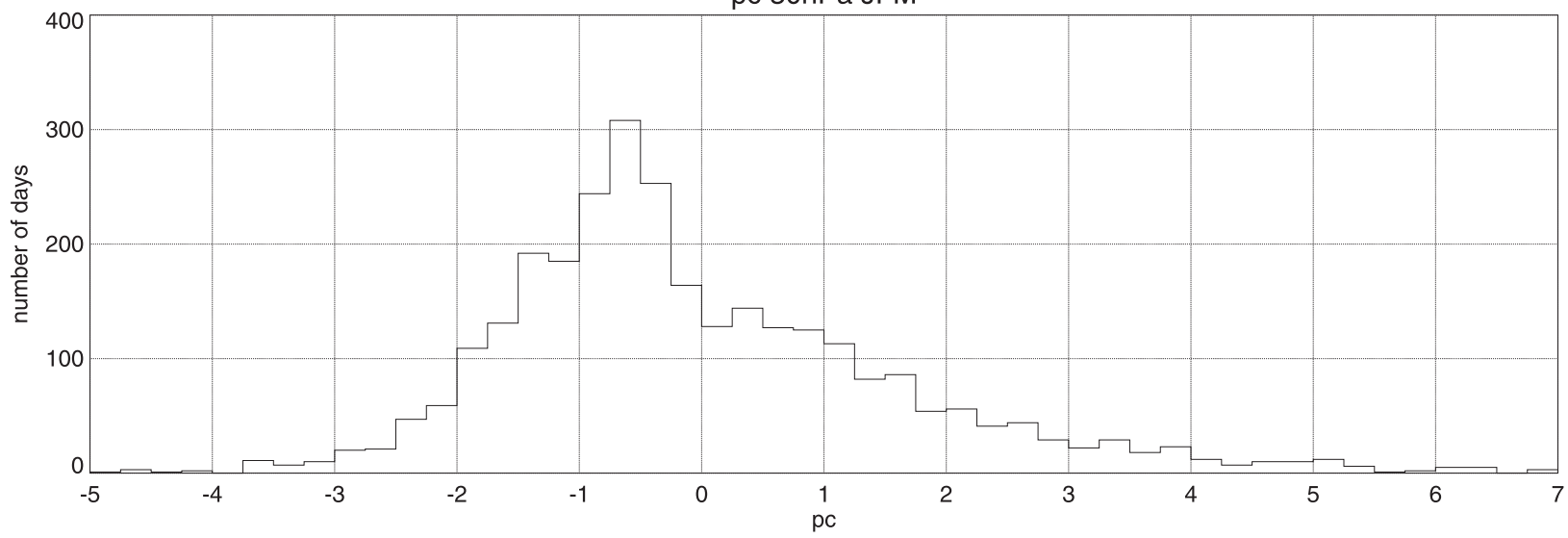

FIG. 1. Climatological zonal-mean wave-1 heat flux during (left) JFM, (middle) the wave-1 heat flux pattern found by regressing the heat flux at all levels on the standardized $30-\mathrm{hPa}$ PC time series, and (right) the total wave- 1 heat flux field for a $-\sigma_{\mathrm{JFM}}=-1.7$ value of the standardized principle component (e.g., the climatological heat flux pattern is added to $-\sigma_{\mathrm{JFM}}$ times the regression pattern). Contour interval is logarithmic in powers of $2: \pm(1,2,4,8,16,32,64,128,256) \mathrm{K} \mathrm{m} \mathrm{s}^{-1}$, negative contours are dashed, and the thick solid line indicates the zero contour. (bottom) Histogram of the daily JFM values of the leading standardized PC at $30 \mathrm{hPa}$.

\section{a. Evolution in the stratosphere}

Figure 2 (top) shows the evolution of the wave- 1 heat flux anomaly projected onto the regression pattern in Fig. 1 (middle) during the composite downward wave coupling event as a function of time from -15 to 15 days and height. Note that the evolution at $30 \mathrm{hPa}$ is equal to the wave coupling index: the leading PC at $30 \mathrm{hPa}$. The shading indicates statistically significant signals at the $95 \%$ level based on a $t$ test. Figure 2 also shows the timeaveraged zonal-mean wave-1 heat flux (middle) and meridional momentum flux (bottom) anomalies during the four stages.

During the first stage the wave coupling index and heat flux anomaly in the stratosphere are positive and statistically significant. The heat flux anomaly maximum moves from 200 to $10 \mathrm{hPa}$ during the stage, indicative of an upward wave coupling precursor. Note that the wave coupling index during this stage is not extreme (it does not exceed $\left.+\sigma_{\mathrm{JFM}}\right)$. The heat flux anomaly in the troposphere and the momentum flux anomaly throughout the atmosphere are not statistically significant during this stage. During the second stage the wave coupling index and heat flux anomaly change sign from positive to negative in the mid to lower stratosphere in high latitudes. In addition, a positive heat flux anomaly develops in the troposphere. The vertical dipole of the heat flux is reminiscent of the regression pattern in Fig. 1 (middle). The momentum flux anomaly exhibits a meridional dipole in the upper stratosphere. During the third stage 
TABLE 1. Central date of downward wave coupling events at $30 \mathrm{hPa}$ and the minimum total 5-day smoothed wave-1 heat flux from $40^{\circ}$ to $90^{\circ} \mathrm{N}$ during the event.

\begin{tabular}{cc}
\hline Date & $\begin{array}{c}\min _{40-90^{\circ} \mathrm{N}} \overline{\boldsymbol{v}^{\prime} T^{\prime}} \\
\left(\mathrm{K} \mathrm{m} \mathrm{s} \mathrm{s}^{-1}\right)\end{array}$ \\
\hline 25 Feb 1979 & -99.37 \\
18 Jan 1984 & -25.54 \\
22 Jan 1986 & -47.30 \\
22 Feb 1989 & -75.81 \\
12 Feb 1990 & -77.66 \\
28 Jan 1991 & -80.75 \\
21 Jan 1992 & -31.94 \\
17 Mar 1993 & -41.18 \\
11 Feb 1995 & -78.63 \\
9 Jan 1996 & -64.24 \\
21 Jan 1997 & -31.71 \\
30 Jan 2005 & -29.27 \\
7 Jan 2007 & -41.99 \\
26 Jan 2008 & -76.00 \\
\hline
\end{tabular}

the wave coupling index decreases significantly in the stratosphere and reaches a minimum value of -3.1 at $30 \mathrm{hPa}$ on day +3 . In addition, there is a statistically significant positive heat flux anomaly in the troposphere. The tropospheric maximum clearly lags the minimum in the stratosphere. The momentum flux anomaly exhibits a vertical dipole with a statistically significant negative signal in the troposphere. Finally during the fourth stage the wave coupling index and heat flux anomaly weaken but remain negative in the stratosphere. The heat flux anomaly in the troposphere weakens significantly and is no longer statistically significant in high latitudes. In contrast, the momentum flux anomaly in the troposphere and stratosphere remain large.

The downward wave coupling event is associated with a transition of the wave coupling index and heat flux anomaly from positive to negative in the stratosphere. Since wave- 1 and the zonal-mean flow are well known to be strongly coupled in the stratosphere, we also consider the evolution of the zonal-mean flow during the life cycle. The structure of zonal-mean flow variability is determined by the leading EOF of zonal-mean geopotential height at each pressure level-for example, the northern annular mode. Recall that the sign of the leading PC of the zonal-mean geopotential height in the stratosphere, called the zonal index, indicates the strength of the stratospheric polar vortex, whereas in the troposphere the zonal index indicates the position of the tropospheric jet. In particular, a positive (negative) index indicates a strong (weak) stratospheric polar vortex and a poleward (equatorward) shift of the tropospheric jet. Because of the strong relationship between the zonal-mean geopotential height and zonal-mean zonal wind, we consider the evolution of both the zonal index, representing the zonal-mean geopotential height, and the zonal-mean zonal wind anomalies during the composite downward wave coupling event. Figure 3 shows the composite evolution of the zonal index (top) and the time-averaged zonal-mean zonal wind anomaly (bottom) during the four stages. Note that the time series of the zonal index at each pressure level is based on the leading PC at that level and is aligned to the central dates of the downward wave coupling events defined by the wave coupling index at $30 \mathrm{hPa}$. The shading indicates statistically significant signals at the $95 \%$ level based on a $t$ test.

During the first stage the zonal index is positive from the lower stratosphere to the surface and negative in the upper stratosphere. In addition, there is a statistically significant positive zonal-mean zonal wind anomaly in the polar lower stratosphere/upper troposphere region and a negative zonal wind anomaly in the subtropical stratosphere. During the second stage the zonal index weakens in the upper stratosphere and reaches a minimum of -1.4 at $1 \mathrm{hPa}$ on day -1 , which is associated with a significant weakening of the polar vortex in the upper stratosphere. In addition, there is a positive zonalmean zonal wind anomaly in high latitudes. Note that the weakening of the zonal index occurs over a relatively short time scale ( $<$ a week) compared to the weakening during extreme weak vortex events, defined by a zonalindex threshold of -3.0 during November to April, which occur over time scales of weeks to months (Baldwin and Dunkerton 2001). During the third stage of the event the zonal index reaches a maximum of 0.9 at $100 \mathrm{hPa}$ on day +11 . The zonal-mean zonal wind anomaly is positive, indicating a strengthening of the polar vortex in the stratosphere and a weakening of the subtropical jet. The negative to positive transition of the zonal index in the upper and lower stratosphere between -5 to +5 days is reminiscent of the zonal-mean geostrophic and hydrostatic adjustment to a potential vorticity anomaly (Black 2002; Ambaum and Hoskins 2002). Finally during the fourth stage the zonal index in the lower stratosphere is positive and coincides with a positive zonal-mean zonal wind anomaly indicating a strengthened polar vortex.

The positive to negative heat flux anomaly transition during the second stage occurs as the zonal index in the upper stratosphere is weakening. To fully understand the evolution of wave- 1 and the zonal-mean flow one must consider total fields. As discussed previously, a negative heat flux anomaly can imply reduced upward wave coupling or downward wave coupling. In particular, reduced upward wave coupling involves a negative anomaly but a positive total heat flux. In contrast, downward wave coupling involves both a negative heat flux anomaly and a negative total heat flux, implying a downward vertical group velocity. Similarly, a negative zonal-mean zonal wind anomaly does not necessarily 

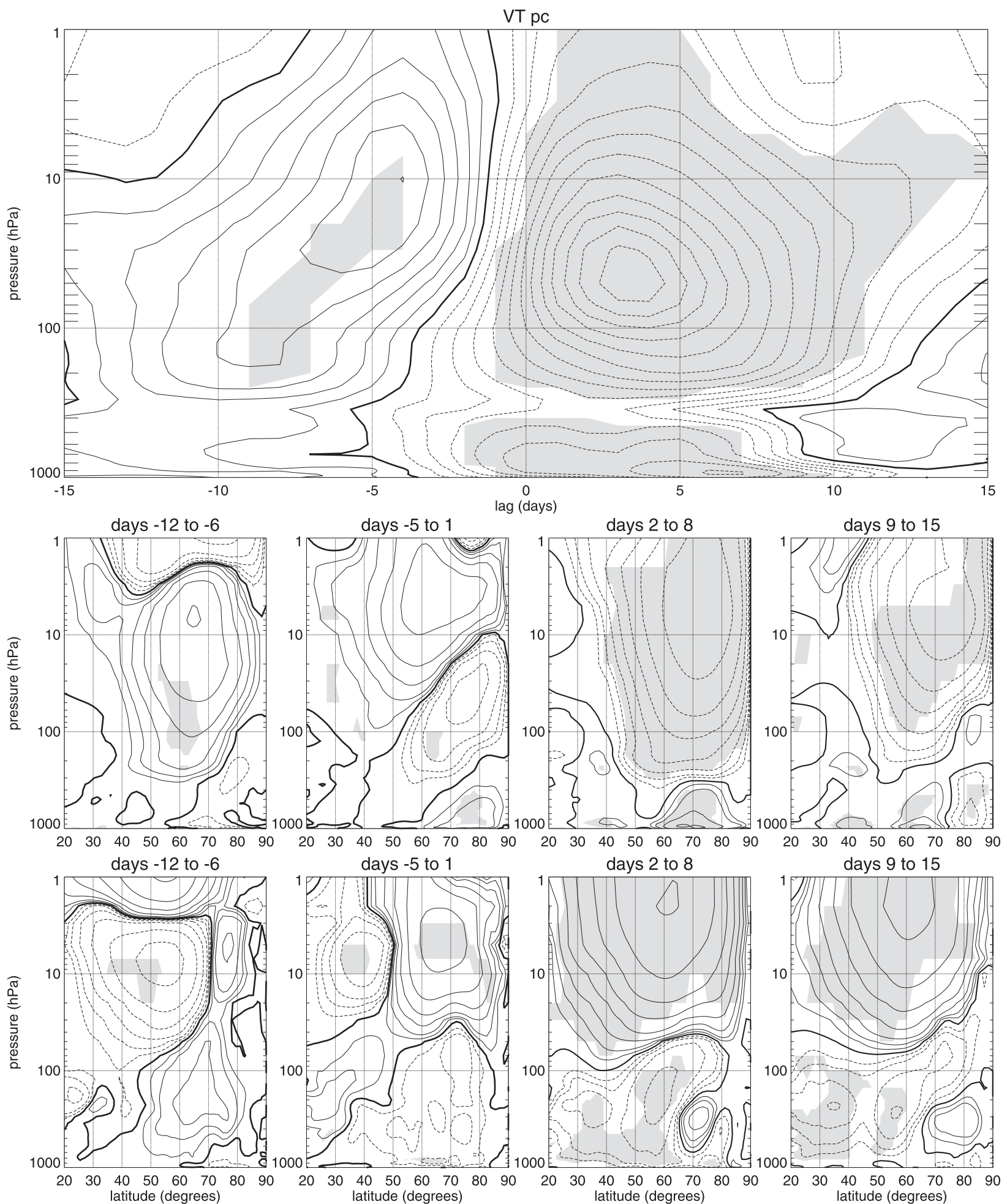

FIG. 2. (top) Evolution of the wave-1 heat flux anomaly projected onto the regression pattern in Fig. 1 (middle panel) for the composite downward wave coupling event as a function of time from -15 to 15 days and height. Contour interval is 0.25 , negative contours are dashed, and the thick solid line indicates the zero contour. (middle row) Anomalous zonal-mean wave-1 meridional heat flux and (bottom row) negative meridional momentum flux averaged from -12 to $-6,-5$ to 1,2 to 8 , and 9 to 15 days. Contour interval as in Fig. 1 for the middle row and equal to $\pm(1,2,4,8,16,32,64,128,256) \mathrm{m}^{2} \mathrm{~s}^{-2}$ for the bottom row. Shading indicates statistical significance at the $95 \%$ level based on a $t$ test. 

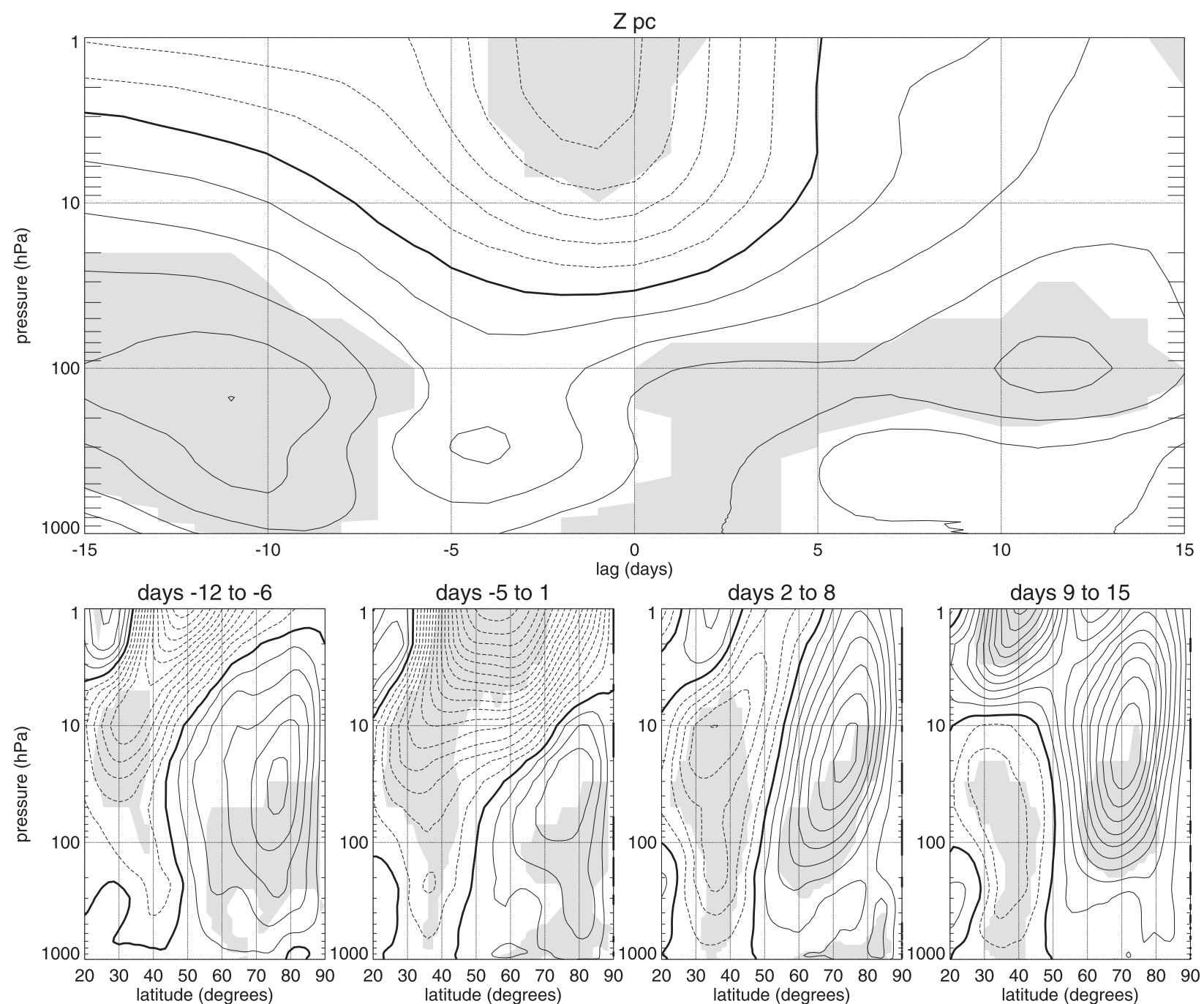

FIG. 3. (top) Evolution of the zonal index (e.g., the leading standardized PC of the zonal-mean geopotential height at each pressure level) for the composite downward wave coupling event as a function of time from -15 to 15 days and height: contouring as in Fig. 2 (top). (bottom row) Anomalous zonal-mean zonal wind averaged from -12 to $-6,-5$ to 1,2 to 8 , and 9 to 15 days: contour interval $1 \mathrm{~m} \mathrm{~s}^{-1}$. Shading indicates statistical significance at the $95 \%$ level based on a $t$ test.

imply that the sign of the zonal-mean zonal wind has changed sign, for example, from westerly to easterly as it does during a sudden stratospheric warming event. The structure of the total zonal-mean zonal wind is also relevant in so far as it acts as a waveguide for wave propagation.

Figure 4 shows the total zonal-mean zonal wind, wave-1 meridional heat flux, negative wave-1 meridional momentum flux, and wave- 1 geopotential height at $70^{\circ} \mathrm{N}$ during the four stages of the composite downward wave coupling event. The shading in the zonal-mean zonal wind panels represents regions of vertical and meridional wave evanescence, defined by negative vertical and meridional wavenumbers. The wavenumbers are calculated from the solution to the wave equation associated with the conservation of potential vorticity in spherical coordinates linearized about a zonal-mean basic state following Harnik and Lindzen (2001). The regions of vertical and meridional wave evanescence indicate regions where wave propagation is not permitted according to linear theory. During the first stage the polar vortex peaks in the upper stratosphere. There is only a small region of vertical wave evanescence in the upper stratosphere, which is associated with a region of negative vertical zonal wind shear. The configuration is very favorable for upward wave coupling as indicated by the positive total heat flux and the westward phase tilt with height from the midtroposphere to the midstratosphere. The wave- 1 amplitude in the midtroposphere is weak, and the pattern at the surface is out of phase with the levels directly above, which is likely because the wave- 1 

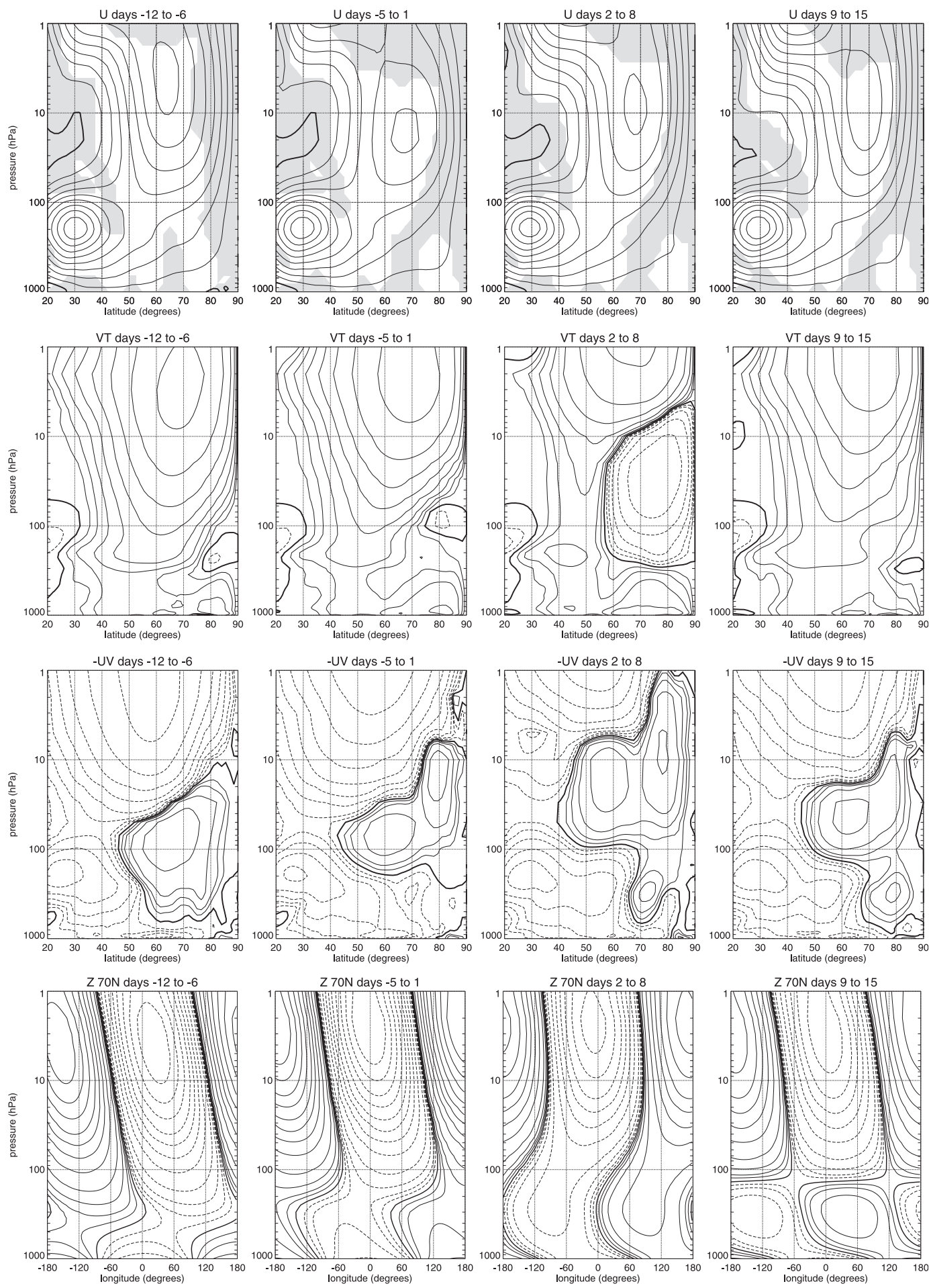

FIG. 4. Evolution of (from top to bottom) the total zonal-mean zonal wind, total wave-1 meridional heat flux, total negative wave- 1 meridional momentum flux, and total wave- 1 geopotential height at $70^{\circ} \mathrm{N}$ for the composite downward wave coupling event averaged from -12 to $-6,-5$ to 1,2 to 8 , and 9 to 15 days. Shading in the top panels indicates regions of wave evanescence. From left to right contour interval is $5 \mathrm{~m} \mathrm{~s}^{-1}$ for the first row, logarithmic in powers of $2, \pm(1,2,4,8,16,32,64,128,256) \mathrm{K} \mathrm{m} \mathrm{s}^{-1}$ in the second row and $\mathrm{m}^{2} \mathrm{~s}^{-2}$ in the third row, and $\pm 1 \times 10^{2}(1,2$, $4,8,16,32,64,128,256) \mathrm{m}$ for the fourth row; Negative contours are dashed, and the thick solid line indicates the zero contour. 

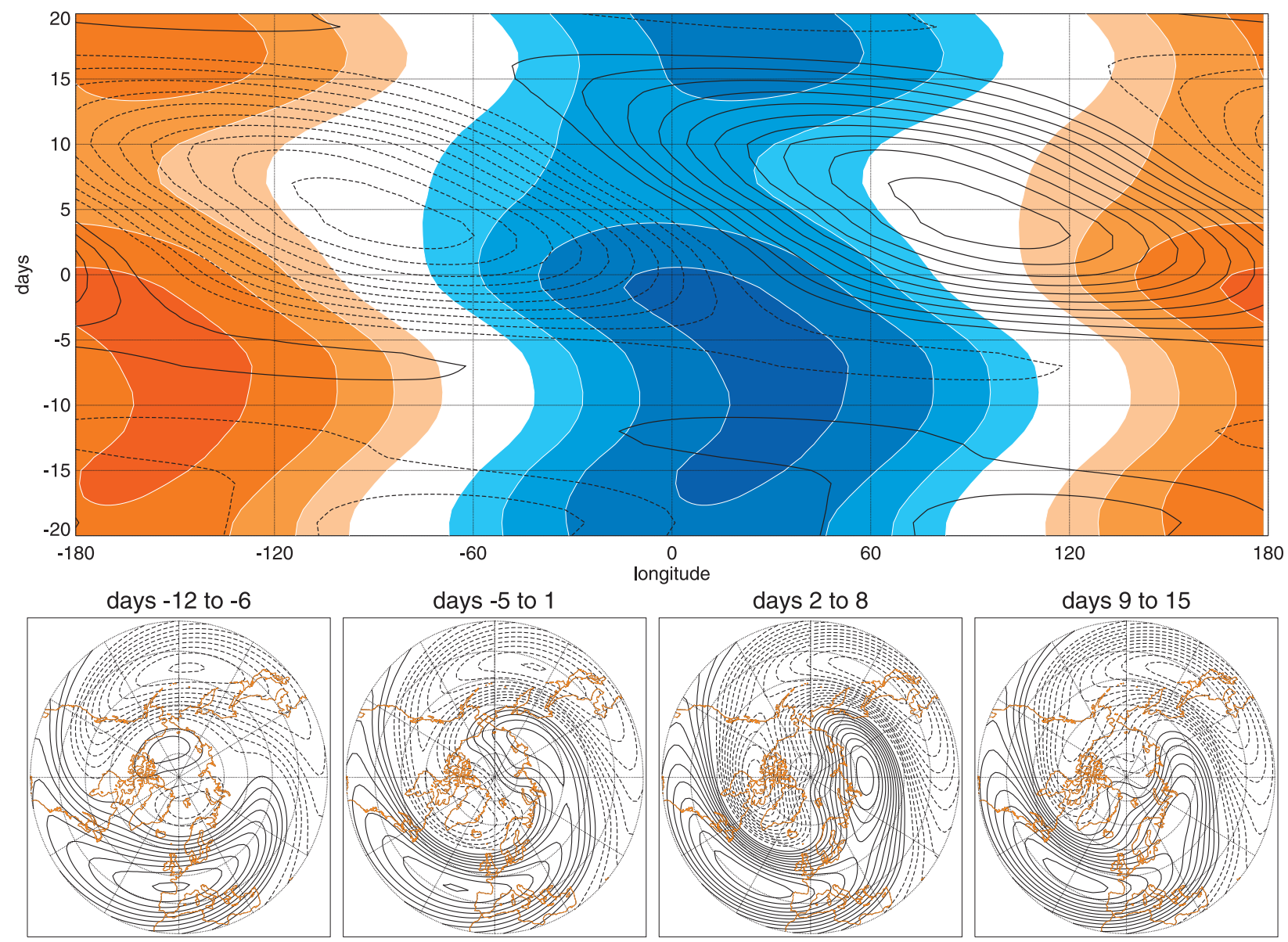

FIG. 5. (top) Evolution of the total 500-hPa (black contours) and $10-\mathrm{hPa}$ (color shading) wave- 1 pattern averaged from $60^{\circ}$ to $80^{\circ} \mathrm{N}$ for the composite downward wave coupling event as a function of time from -20 to 20 days and longitude: Contour interval $20 \mathrm{~m}$ (color shading) and $10 \mathrm{~m}$ (black contours). (bottom) The total 500-hPa wave- 1 averaged from -12 to $-6,-5$ to 1,2 to 8 , and 9 to 15 days: contour interval $10 \mathrm{~m}$.

pattern at the surface is strongly constrained by orography and ocean-land heating asymmetries. The momentum flux is poleward in the high-latitude upper troposphere/lower stratosphere and equatorward in the upper stratosphere.

During the second stage the maximum zonal-mean zonal wind resides just below $10 \mathrm{hPa}$ and consequently the region of negative vertical zonal wind shear and vertical wave evanescence extends down to $5 \mathrm{hPa}$. The descent of the wave evanescence region seems to coincide with the upward wave coupling precursor. In addition, the latitudinal width of the meridional waveguide becomes narrower during this stage. Overall, the vortex configuration is very favorable for downward wave coupling because upward propagating waves will almost certainly encounter the reflecting surface and will be forced to reflect and propagate downward into the troposphere because of the narrow meridional waveguide. There are signs of the beginning stages of wave reflection in the lower stratosphere where the total heat flux is negative and the westward phase tilt from the lower to upper stratosphere has weakened. The wave-1 amplitude in the midtroposphere has increased and at the surface appears to extend into the free troposphere; however, this is partly the result of the expansion of the wave- 1 pattern in high latitudes, which cannot be captured by showing the wave pattern at a single latitude (see Fig. 5, bottom).

During the third stage the zonal-mean zonal wind configuration remains favorable for wave reflection. The total wave- 1 heat flux is negative in the polar upper troposphere/lower stratosphere to the midstratosphere and its magnitude is as large as the positive heat flux during stage one. In addition, the vertical Eliassen-Palm flux is negative in the high-latitude mid to lower stratosphere (not shown) while its vertical divergence is positive (not shown). The divergence of Eliassen-Palm flux is related to the transient evolution of the wave activity. The 
region of negative momentum flux expands poleward suggesting enhanced equatorward propagation in midlatitudes during this stage (cf. with Fig. 2, bottom). The wave pattern exhibits a clear eastward phase tilt with height from the midtroposphere to the midstratosphere, which is indicative of wave reflection and downward wave coupling. The whole wave pattern from the midtroposphere to midstratosphere has shifted westward, and the wave pattern in the midtroposphere reaches its maximum amplitude. Finally during the fourth stage the zonal-mean zonal wind has strengthened in the mid and lower troposphere. The heat flux is positive throughout the atmosphere and wave 1 exhibits a clear standing wave pattern in the vertical. The standing wave pattern implies interference between upward and downward propagating waves. The sign of the heat flux suggests that the upward propagating wave dominates.

\section{b. Impact on the troposphere}

Downward wave- 1 coupling events clearly coincide with changes in the tropospheric wave pattern. In particular, there is a pronounced amplification and westward phase shift of the high-latitude wave-1 pattern that occurs as it achieves an eastward phase tilt with height from the midtroposphere to the midstratosphere (see Fig. 4, bottom). The evolution of the high-latitude wave-1 pattern can be illustrated using a Hovmöller plot. Figure 5 (top) shows the total wave- 1 pattern averaged from $60^{\circ}$ to $80^{\circ} \mathrm{N}$ at $500 \mathrm{hPa}$ (black contours) and $10 \mathrm{hPa}$ (color) as a function of longitude and time from -15 to +15 days. The longitude-latitude patterns from $30^{\circ}$ to $90^{\circ} \mathrm{N}$ at $500 \mathrm{hPa}$ during the four stages are also shown (Fig. 5, bottom). During the first stage the 500-hPa highlatitude wave pattern is very weak, consistent with the climatological pattern at these latitudes. Note, however, that there is a robust wave- 1 pattern in midlatitudes that is out of phase with the high-latitude wave-1 pattern. During the second stage the amplitude of the $10-\mathrm{hPa}$ high-latitude wave pattern reaches a maximum and precedes the maximum amplitude at $500 \mathrm{hPa}$, which occurs during stage three. The phase tilt with height from 500 and $10 \mathrm{hPa}$ is clearly eastward. The midlatitude wave- 1 pattern at $500 \mathrm{hPa}$ does not exhibit a large change in amplitude or phase. During this stage the phase of the high-latitude wave-1 pattern at $500 \mathrm{hPa}$ begins to shift westward, which is consistent with the pattern being synchronized with the levels above. The amplitude of the high-latitude 500-hPa wave pattern reaches a maximum during the third stage. At the same time the pattern continues to move westward and by the end of the stage the phase has moved approximately $120^{\circ}$ wesward, which implies a phase speed of approximately $-2.6 \mathrm{~m} \mathrm{~s}^{-1}$. During this stage the high-latitude wave pattern is clearly out of phase with the midlatitude wave pattern. Finally, during the fourth stage the amplitude of the high latitude wave- 1 pattern decreases and the midlatitude pattern is strengthened consistent with equatorward wave propagation. Overall the wave pattern evolution is very consistent with a downward wave coupling event: a stratospheric wave-1 anomaly at $10 \mathrm{hPa}$ precedes a tropospheric wave- 1 anomaly at $500 \mathrm{hPa}$ and the wave- 1 pattern exhibits an eastward phase tilt with height.

Downward wave coupling clearly impacts both the wave-1 amplitude and phase in the midtroposphere. Since baroclinic scale wavenumbers also contribute to the geopotential height at $500 \mathrm{hPa}$, we consider the evolution of the full height anomaly field. Figure 6 (top) shows the evolution of 500-hPa geopotential height anomaly averaged from $90^{\circ} \mathrm{W}$ to $40^{\circ} \mathrm{E}$ as a function of latitude and time from -15 to +15 days during the downward wave coupling event together with the longitude-latitude patterns from $30^{\circ}$ to $90^{\circ} \mathrm{N}$ during the four stages (bottom). During the first stage the geopotential height anomaly involves a high wavenumber and is relatively weak. In the Atlantic sector there is only a small region of statistical significance near the pole. As the event evolves into the second stage, a wave- 1 signal develops. The negative wave- 1 anomaly is centered at $30^{\circ} \mathrm{W}$ with the positive lobe at $150^{\circ} \mathrm{E}$ and coincides with the high-latitude wave- 1 anomaly (see Fig. 5). As a result there is a large negative anomaly in the Atlantic region between $60^{\circ}$ and $70^{\circ} \mathrm{N}$. During the third stage the geopotential height anomaly exhibits a statistically significant wave-1 pattern poleward of $50^{\circ} \mathrm{N}$, and the phase of the pattern is identical to the wave-1 pattern shown in Fig. 5. In addition, a positive anomaly develops in midlatitudes in the Atlantic region consistent with equatorward wave propagation. The positive anomaly clearly lags the negative anomaly from the second stage and, in combination, they resemble the positive phase of the North Atlantic Oscillation (NAO). The NAO is the leading mode of variability in the North Atlantic region and represents a pressure seesaw between mid and high latitudes that reflects the position of the tropospheric jet over the North Atlantic (Hurrell et al. 2003). Finally, during the fourth stage the geopotential height anomalies in the Atlantic sector weaken significantly.

The NAO is associated with well-known weather regimes. In particular, during the positive phase there is a near-surface negative temperature anomaly over northeastern North America and a positive anomaly over western Eurasia (Hurrell et al. 2003). Figure 7 (top) shows the evolution of the $850-\mathrm{hPa}$ temperature anomaly averaged from $60^{\circ}$ to $80^{\circ} \mathrm{N}$ as a function of longitude and time from -15 to +15 days during the downward wave coupling event together with the longitude-latitude patterns from $30^{\circ}$ to $90^{\circ} \mathrm{N}$ during the four stages (bottom). Note that the 


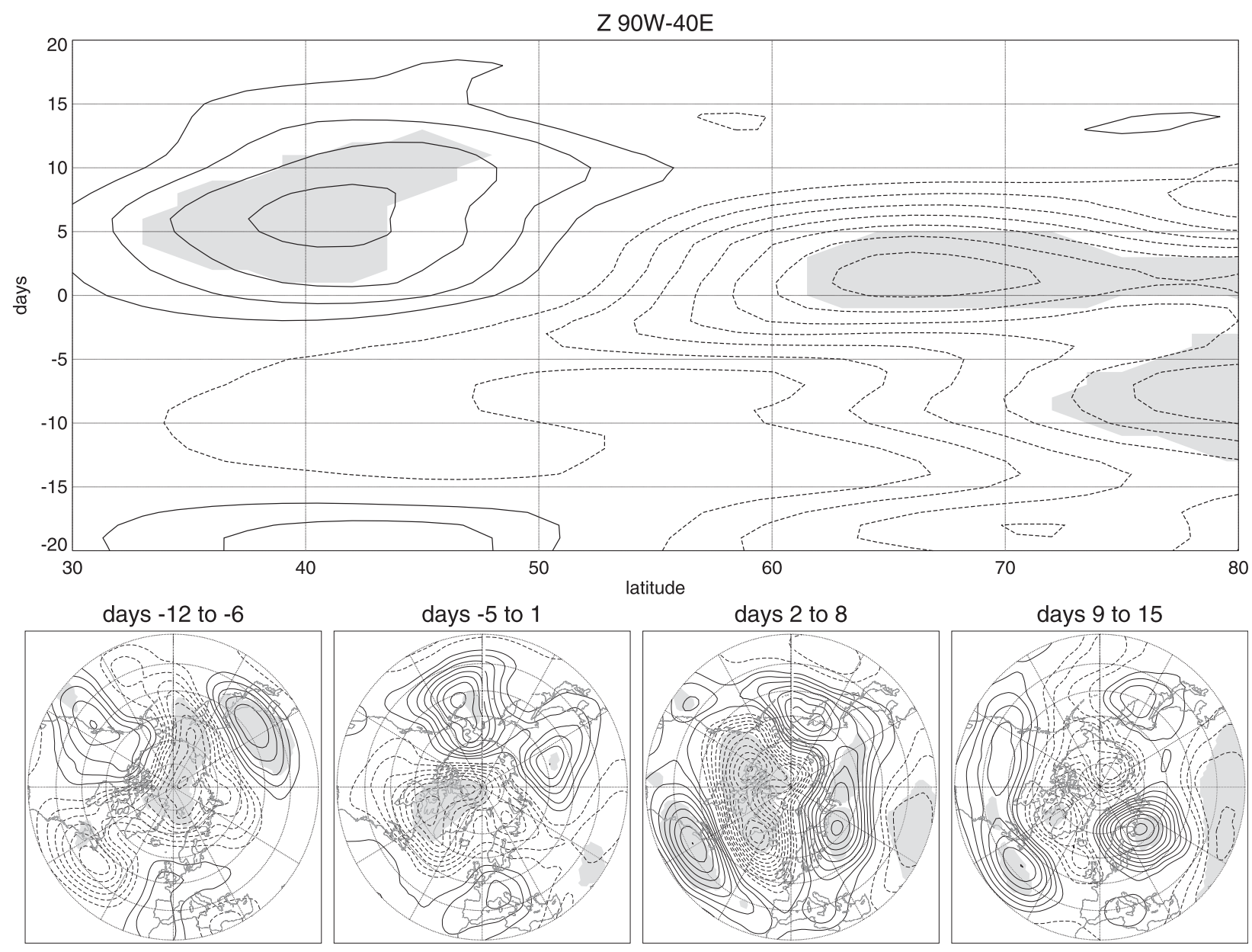

FIG. 6. (top) Evolution of the 500-hPa geopotential height anomaly averaged from $90^{\circ} \mathrm{W}$ to $40^{\circ} \mathrm{E}$ for the composite downward wave coupling event as a function of time from -20 to 20 days and latitude. (bottom) The 500-hPa geopotential height anomaly averaged from -12 to $-6,-5$ to 1,2 to 8 , and 9 to 15 days: contour interval $10 \mathrm{~m}$. Shading indicates statistical significance at the $95 \%$ level based on a $t$ test.

near-surface $(2 \mathrm{~m})$ temperature evolution closely resembles the $850-\mathrm{hPa}$ temperature (not shown). During the first stage the anomalies are weak, and there is only a small region of statistical significance near the pole. As the event proceeds through the second stage, a negative anomaly appears over northeastern North America and a positive anomaly appears over northern Eurasia. The negative anomalies increase and peak at the beginning of the third stage, and their location is consistent with the negative wave-1 lobe over the Atlantic region. In contrast, the positive anomalies reach a maximum at the end of the third stage. The evolution of the anomalies is consistent with advection of cold and warm air related to the anomalous flow associated with the wave- 1 pattern over the Atlantic region. Finally, during the fourth stage the wave- 1 temperature pattern in the high-latitude region weakens, while the anomalies in the midlatitudes remain large.
The evolution of the $850-\mathrm{hPa}$ zonal wind anomaly averaged from $90^{\circ} \mathrm{W}$ to $40^{\circ} \mathrm{E}$ as a function of latitude and time from -15 to +15 days during the downward wave coupling event is shown in Fig. 8 (top). The longitudelatitude patterns from $30^{\circ}$ to $90^{\circ} \mathrm{N}$ during the four stages are also shown in Fig. 8 (bottom). As for the $850-\mathrm{hPa}$ temperature anomaly, the largest impacts occur during the third stage when there is a clear wave- 1 signature in high latitudes. In addition there is a clear poleward jet shift in the Atlantic region, which persists during stage four.

Finally, the evolution of mean sea level pressure anomaly averaged from $90^{\circ} \mathrm{W}$ to $40^{\circ} \mathrm{E}$ as a function of longitude and time from -15 to +15 days is shown in Fig. 9 (top). The longitude-latitude patterns from $30^{\circ}$ to $90^{\circ} \mathrm{N}$ during the four stages are also shown in Fig. 9 (bottom). During the first stage the anomalies are weak and exhibit a high wavenumber similar to the $850-\mathrm{hPa}$ 


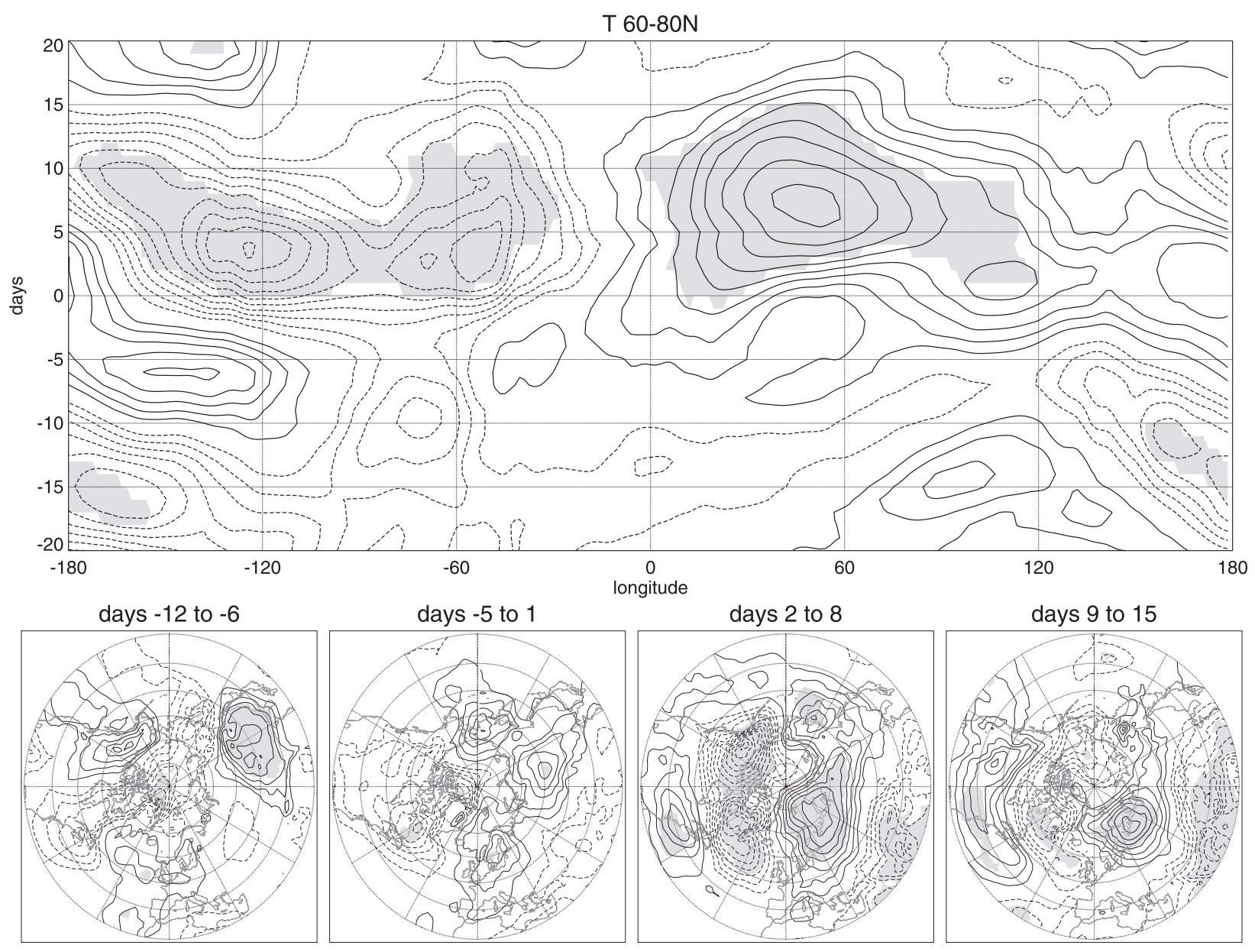

FIG. 7. Evolution of (top) the 850-hPa temperature anomaly averaged from $60^{\circ}$ to $80^{\circ} \mathrm{N}$ for the composite downward wave coupling event as a function of time from -20 to 20 days and longitude. (bottom) The anomalous 850 -hPa temperature averaged from -12 to -6 , -5 to 1,2 to 8 , and 9 to 15 days: contour interval $0.5 \mathrm{~K}$. Shading indicates statistical significance at the $95 \%$ level based on a $t$ test.

temperature anomalies. During the second stage a negative mean sea level pressure anomaly develops in the Atlantic sector and reaches its minimum. The negative anomaly is shifted slightly eastward relative to the geopotential height anomaly aloft. In addition, the mean sea level pressure anomaly is consistent with the zonal index during this stage (see Fig. 3). A positive anomaly appears in midlatitudes during the third stage consistent with the positive NAO phase aloft. During the fourth stage the anomalies weaken significantly.

\section{c. Seasonal impact}

An individual downward wave coupling event has a statistically significant impact on the troposphere. The event occurs over a 28-day period with the impact in the troposphere focused during a 10-day period. While an individual event impacts the troposphere on a weekly time scale, several events may occur during an individual JFM season and thereby produce an impact on longer time scales. Here we consider the cumulative impacts in the troposphere during JFM seasons that are dominated by downward wave coupling between the stratosphere and the troposphere. In addition, we consider how the impacts differ from those during weak and strong vortex seasons, which have been studied extensively during recent years (e.g., Baldwin and Dunkerton 1999, 2001; Baldwin et al. 2003; Polvani and Waugh 2004).

The seasonal impact of wave- 1 coupling is assessed using the wave coupling index summed over JFM days. Figure 10 shows the time series of the sum of the wave coupling index during JFM from 1979 to $2011 .^{1}$ The asterisks (open squares) indicate the eight years when the index exceeds $-0.25(+0.25)$ standard deviations and represent years with large downward (upward) wave coupling. The $500-\mathrm{hPa}$ wave- 1 anomaly, the $500-\mathrm{hPa}$

\footnotetext{
${ }^{1}$ Similar impacts are seen for the December-March seasons.
} 

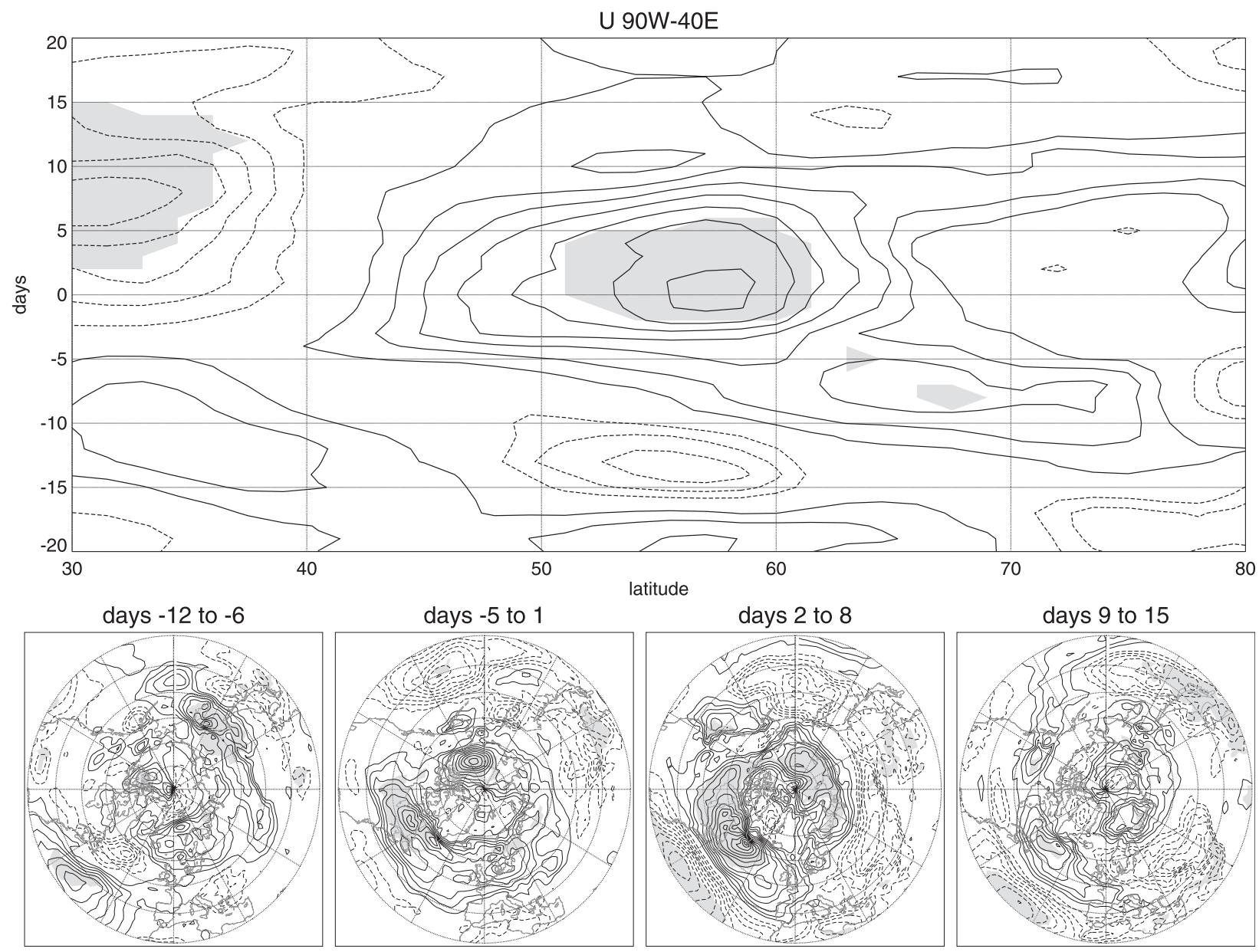

FIG. 8. Evolution of (top) the 850-hPa zonal wind anomaly averaged from $90^{\circ} \mathrm{W}$ to $40^{\circ} \mathrm{E}$ for the composite downward wave coupling event as a function of time from -20 to 20 days and latitude. (bottom) The anomalous 850 -hPa zonal wind averaged from -12 to $-6,-5$ to 1,2 to 8 , and 9 to 15 days: contour interval $0.5 \mathrm{~m} \mathrm{~s}^{-1}$. Shading indicates statistical significance at the $95 \%$ level based on a $t$ test.

geopotential height anomaly, the 850 -hPa temperature anomaly, and the mean sea level pressure anomaly during the years indicated by asterisks and open squares are shown in Fig. 10 (middle and bottom, respectively). During years with a large negative wave coupling index and, hence, large downward wave coupling, the amplitude of the high-latitude total wave-1 pattern is large and dominated by the wave- 1 anomaly (not shown). The highlatitude geopotential height anomaly at $500 \mathrm{hPa}$ is clearly dominated by the wave- 1 anomaly. In addition, there is cooling over northeastern North America and warming over northwestern Eurasia. Finally, the mean sea level pressure anomaly is negative in high latitudes in the Atlantic region with a positive anomaly in midlatitudes. All of these features are consistent with the positive phase of the NAO and were seen for the composite downward wave coupling event discussed in section $3 b$. Note that there are large signals in the North Pacific not seen in the individual event that likely reflect the potential impact of
El Niño-Southern Oscillation events, which do not average out because of the small sample size.

During years with a large positive wave coupling index and hence large upward wave coupling, the total and anomaly wave-1 pattern in high latitudes are very weak. The geopotential height anomaly in the Atlantic sector is weak and exhibits a pattern consistent with the negative phase of the NAO. The temperature anomaly involves warming over North America and cooling over Eurasia, and the mean sea level pressure anomaly clearly reflects the geopotential height pattern at $500 \mathrm{hPa}$. Overall the response during years with a large positive wave coupling index are opposite in sign to years with a large negative value: that is, they are consistent with the negative phase of the NAO; however, the anomalies for positive years are much weaker.

As discussed in the introduction, a standard paradigm of the impact of the stratosphere on the troposphere is via weak/strong stratospheric polar vortex events, (e.g., 


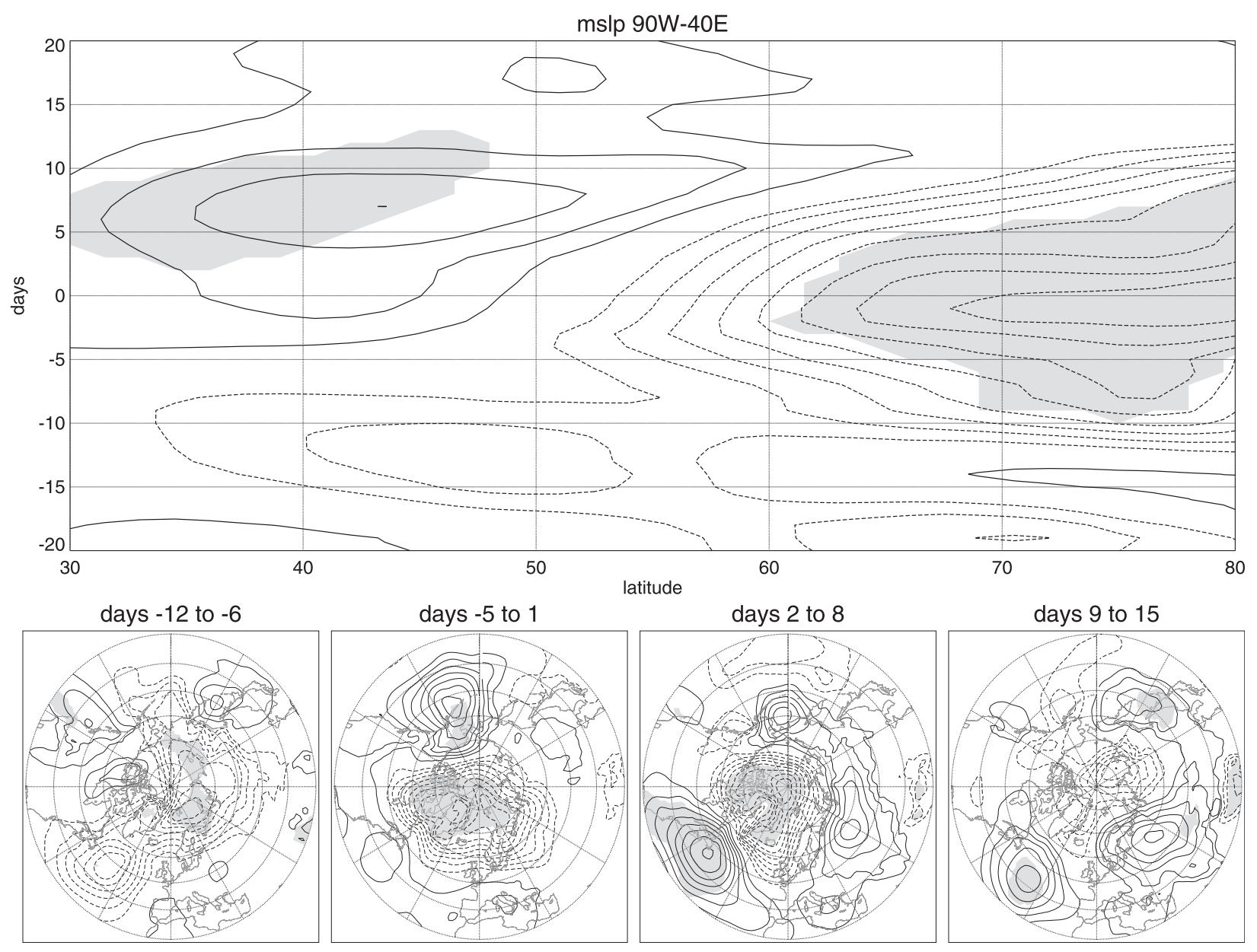

FIG. 9. Evolution of (top) the mean sea level pressure anomaly averaged from $90^{\circ} \mathrm{W}$ to $40^{\circ} \mathrm{E}$ for the composite downward wave coupling event as a function of time from -20 to 20 days and latitude. (bottom) The anomalous mean sea level pressure averaged from -12 to $-6,-5$ to 1,2 to 8 , and 9 to 15 days: contour interval $1 \mathrm{hPa}$. Shading indicates statistical significance at the $95 \%$ level based on a $t$ test.

Baldwin and Dunkerton 2001; Polvani and Waugh 2004). Therefore, we compare the seasonal composites of the wave coupling index extremes with seasonal composites based on extremes of the zonal index. Figure 11 shows the time series of the average zonal index at $30 \mathrm{hPa}$ (the $\mathrm{PC}$ of the zonal-mean geopotential at $30 \mathrm{hPa}$ ) during JFM from 1979 to 2011 . $^{2}$ The asterisks (open squares) indicate the eight years when the average zonal index exceeds $-0.25(+0.25)$ standard deviations. Recall that the zonal index indicates the strength of the polar vortex. During years with a large negative zonal index and hence a weak polar vortex, there is a large high-latitude wave- 1 anomaly that dominates the 500-hPa geopotential height anomaly. The 850 -hPa temperature

\footnotetext{
${ }^{2}$ Similar impacts occur for a zonal index defined at $10 \mathrm{hPa}$ and for the December-March seasons.
}

anomaly involves warming over northern North America and cooling over Eurasia. The mean sea level pressure anomaly is shifted eastward relative to the anomaly at $500 \mathrm{hPa}$. Overall the patterns are consistent with the negative phase of the NAO. Note that only one of the eight weak vortex years corresponds with years with strong upward wave coupling (cf. squares in Fig. 10 to stars in Fig. 11).

During years with a positive zonal index and hence a strong polar vortex, the high-latitude total and anomaly wave- 1 pattern at $500 \mathrm{hPa}$ are weaker, and their sign and structure are reminiscent of the patterns seen during years with a large negative wave coupling index (see Fig. 10). The high-latitude geopotential height at $500 \mathrm{hPa}$, the temperature at $850 \mathrm{hPa}$, and the mean sea level pressure anomalies are all much weaker than during years with a weak vortex. The mean sea level pressure anomaly displays a significant zonal structure. Overall, the patterns 
cumulative wave-coupling index JFM

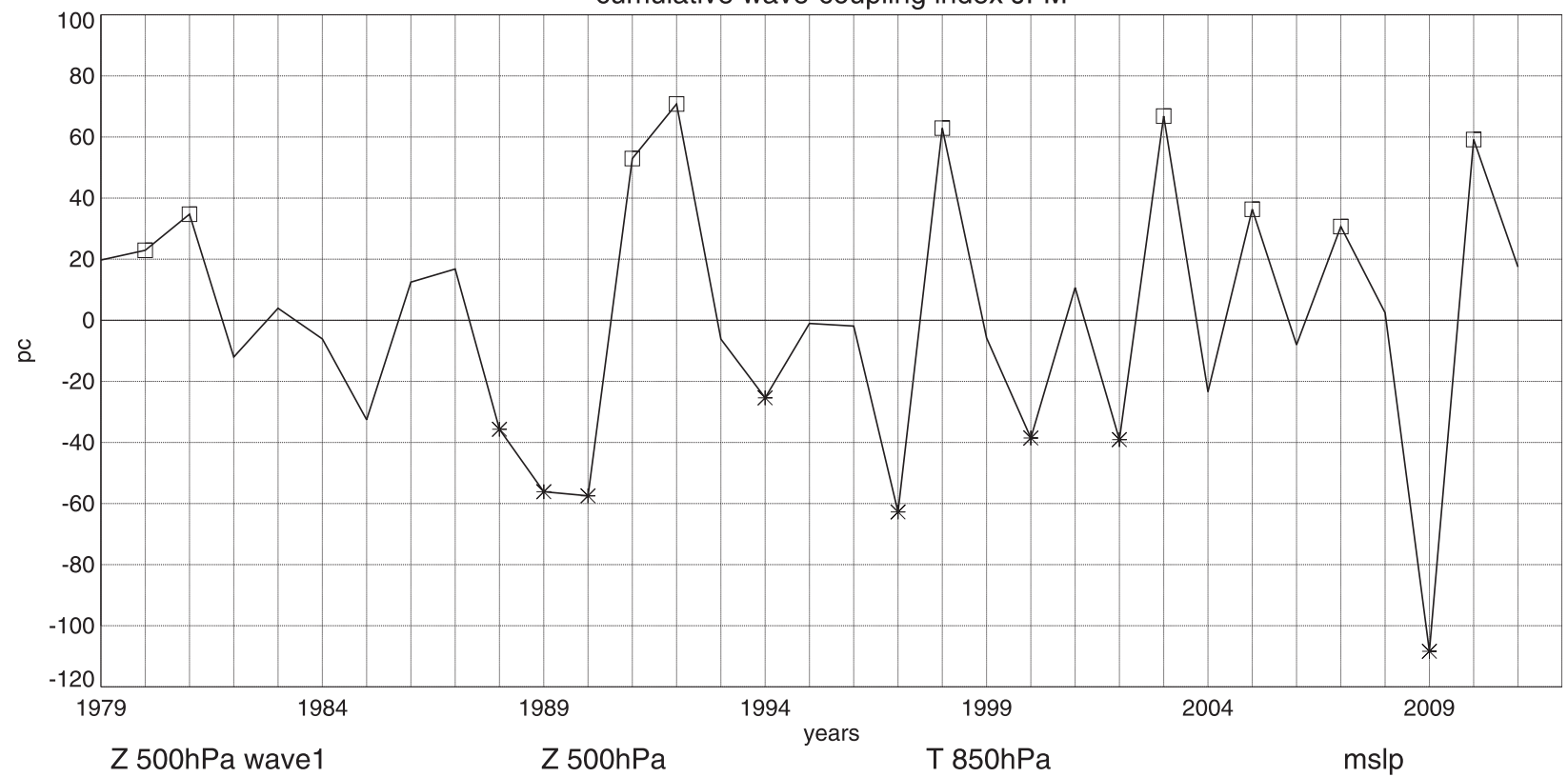

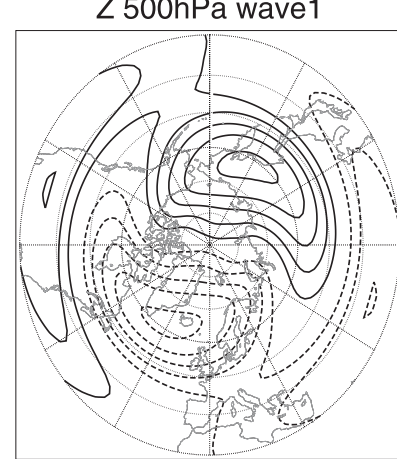

Z 500hPa wave1

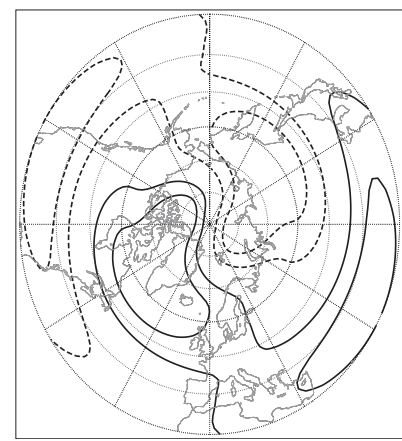

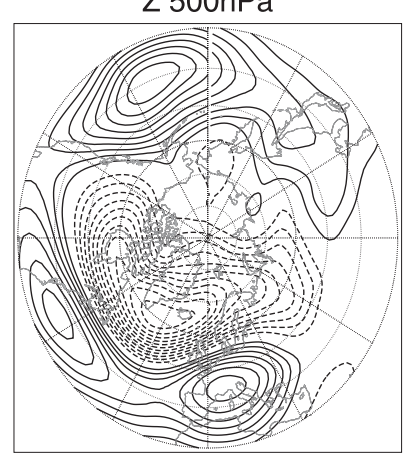

$\mathrm{Z} 500 \mathrm{hPa}$

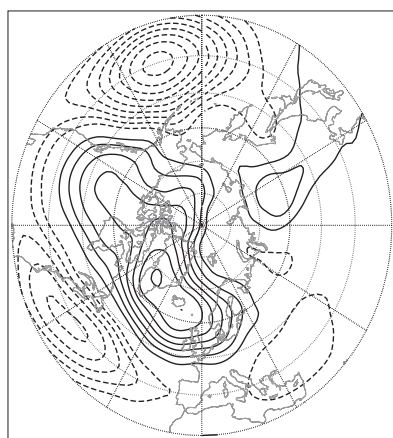

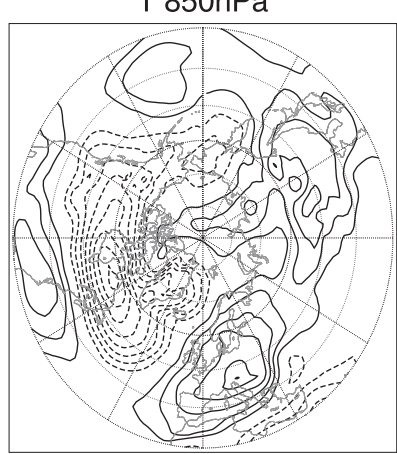

$\mathrm{T} 850 \mathrm{hPa}$

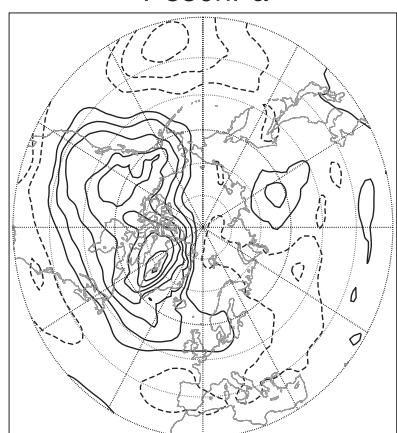

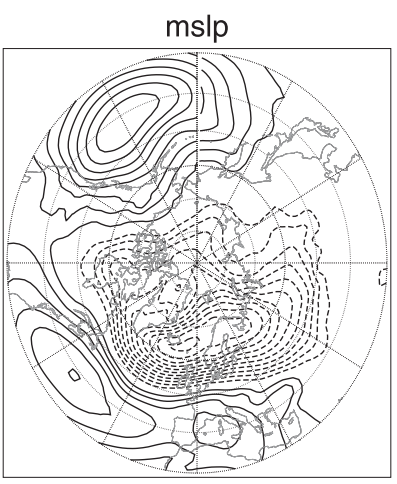

mslp

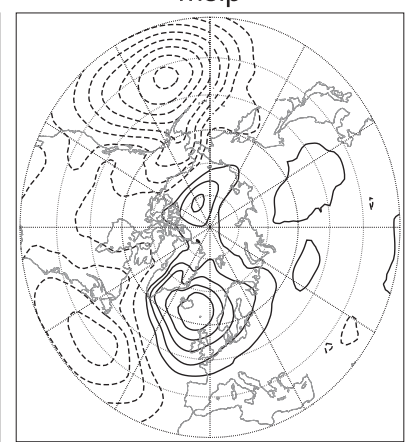

FIG. 10. (top) Sum of the JFM wave coupling index (leading PC of the wave-1 flux at $30 \mathrm{hPa}$ ) as a function of year: (middle row) the 500-hPa wave-1, the 500-hPa geopotential height, the $850-\mathrm{hPa}$ temperature, and the mean sea level pressure anomalies during years with an asterisk. (bottom row) As above but for years with an open square. Contour interval is $5 \mathrm{~m}$ for 500 -hPa wave- 1 and 500 -hPa geopotential height anomalies, $0.25 \mathrm{~K}$ for $850-\mathrm{hPa}$ temperature anomaly, and $0.5 \mathrm{hPa}$ for mean sea level pressure anomaly.

are consistent with the positive phase of the NAO. Note that four out of the eight strong vortex years coincide with downward wave coupling years (cf. asterisks in Fig. 10 to open squares in Fig. 11). The tropospheric response during winter seasons with large downward wave coupling is larger than during years with a strong vortex (by a factor of 2). The composites suggest that the largest impact in the troposphere occurs during seasons with large downward wave coupling or a weak polar vortex, which correspond to the negative tails of the wave coupling and zonal index histograms. The tropospheric impacts are associated with positive and negative phases of the NAO, respectively. 
average zonal index JFM

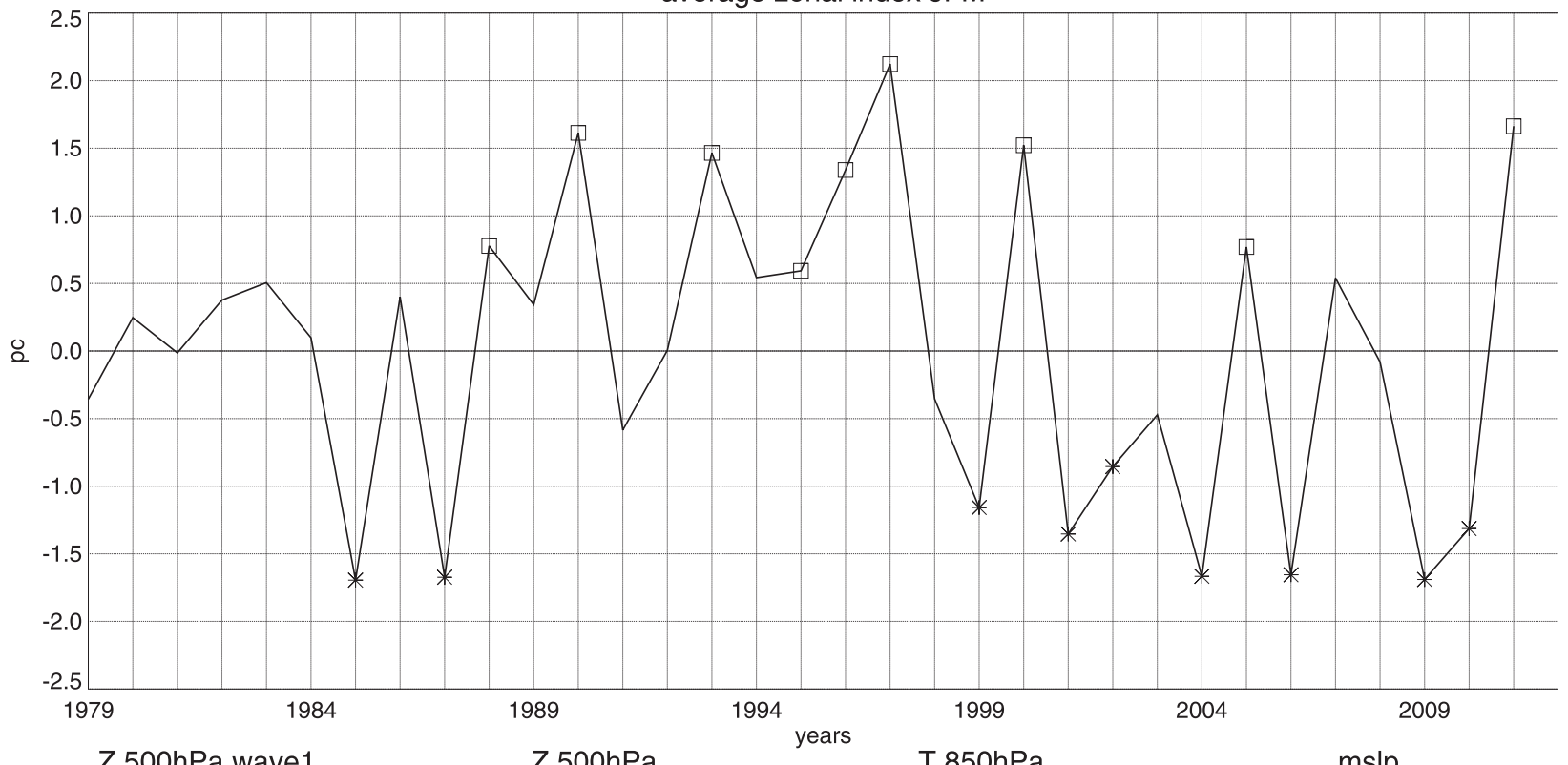

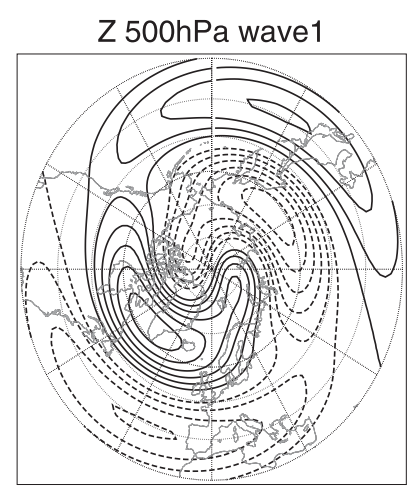

Z 500hPa wave1

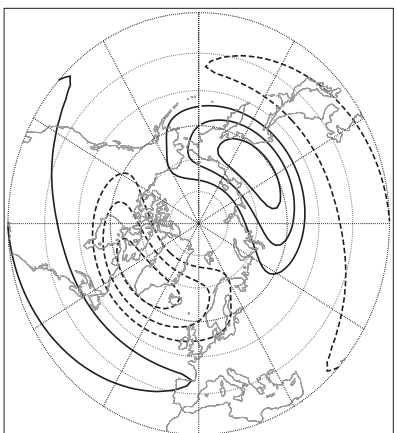

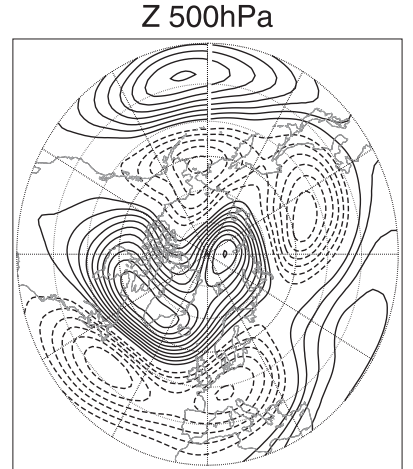

Z 500hPa

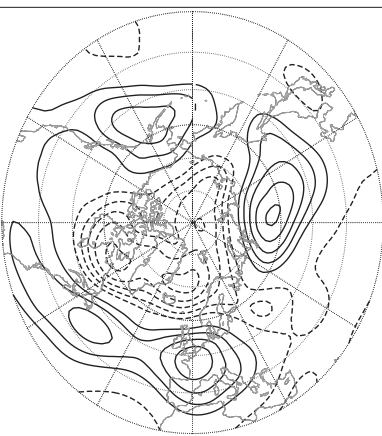

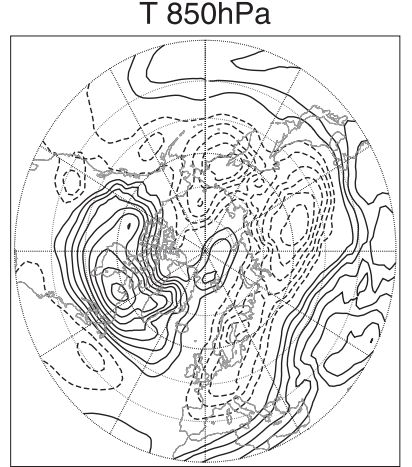

$\mathrm{T} 850 \mathrm{hPa}$

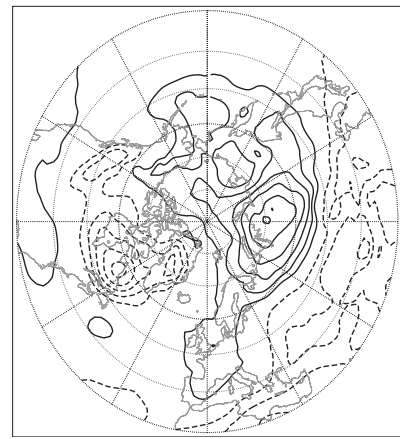

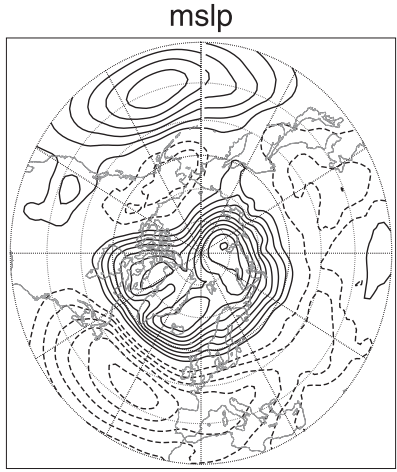

mslp

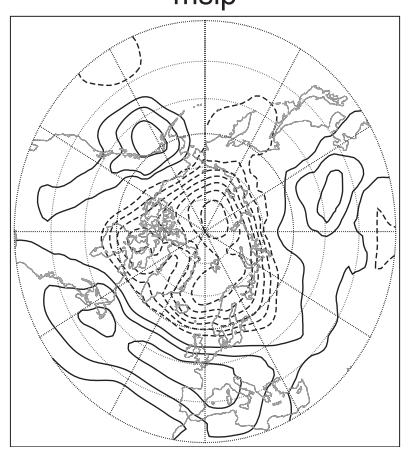

FIG. 11. (top) The JFM average zonal index (leading PC of the zonal-mean geopotential at $30 \mathrm{hPa}$ ) as a function of year: (middle row) The 500-hPa wave-1, the 500-hPa geopotential height, the $850-\mathrm{hPa}$ temperature, and the mean sea level pressure anomalies during years with an asterisk. (bottom row) As above, but for years with an open square. Contouring as in Fig. 10.

\section{Summary and discussion}

The life cycle of Northern Hemisphere downward wave coupling (wave reflection) between the stratosphere and troposphere is analyzed using a composite approach. Downward wave coupling events are isolated as extreme negative heat flux events using a daily wave coupling index defined as the leading PC of the wave- 1 heat flux at $30 \mathrm{hPa}$. The life cycle of the events spans a 28-day period.

The results illustrate that downward wave coupling from the stratosphere to the troposphere involves 
large changes of the wave- 1 pattern and basic state in the stratosphere. The event begins with a positive heat flux precursor in the stratosphere and a wave-1 pattern that exhibits a clear westward phase tilt from the midtroposphere to the midstratosphere, indicating upward wave coupling. In addition, the polar vortex is conducive to upward wave propagation. A subsequent weakening of the polar vortex in the upper stratosphere produces a region of negative vertical zonal wind shear and therefore a region of vertical wave evanescence that acts as a vertical reflecting surface. The high-latitude wave-1 structure subsequently exhibits a clear eastward phase tilt from the midtroposphere to the upper stratosphere, indicating wave reflection and downward wave coupling. In addition, the total heat flux and vertical Eliassen-Palm flux are negative in the polar mid to lower stratosphere and the vertical flux divergence is positive. After the event, wave- 1 exhibits a clear standing wave pattern and the polar vortex is strengthened.

Overall, the evolution in the stratosphere is very consistent with previous results by SPH10, Perlwitz and Harnik $(2003,2004)$ who investigated downward wave coupling on interseasonal and interannual time scales. In particular, the time scale of downward wave coupling events, the zonal-mean zonal wind configuration, and the wave- 1 pattern are all consistent with previous results. The life cycle of downward wave coupling events suggests that the upward propagating precursor plays an important role in the evolution of the zonalmean zonal wind and the formation of the reflecting surface. The importance of the upward precursor was also highlighted by Harnik (2009) who showed that wave reflection is associated with short time scale pulses of upward wave activity from the troposphere that produces a short time scale deceleration of the zonalmean zonal wind and the subsequent reflection of the remaining wave activity. In addition the results suggest that downward wave coupling events modify the wavedriven residual (Brewer-Dobson) circulation via changes in wave- 1 heat flux in the stratosphere. The detailed impact of the events on the circulation will be reported elsewhere.

The current study quantifies for the first time the impact of downward wave coupling on the troposphere. The impact in the troposphere is associated with a transition over a 10-day period toward a positive phase of the NAO and follows the wave reflection event in the stratosphere. The NAO signal develops as a result of a large amplitude high-latitude wave-1 anomaly at $500 \mathrm{hPa}$ that exhibits a clear westward phase progression and is directly coupled to the wave- 1 evolution at $10 \mathrm{hPa}$. The negative lobe of the high-latitude wave- 1 anomaly at $500 \mathrm{hPa}$ is followed by a positive anomaly in midlatitudes due to equatorward propagation that together produces a positive NAO pattern. The impacts on near-surface circulation and temperature during the event are consistent with those observed during the positive phase of the NAO. The adjustment of the polar vortex to the negative heat flux forcing also contributes to the mean sea level pressure anomaly.

The impacts in the troposphere are in agreement with previous statistical results by Perlwitz and Graf (1995) who highlighted a nonzonal connection between the stratosphere and the tropospheric geopotential height that is most pronounced in the Atlantic region. The mean sea level pressure anomalies associated with the vortex adjustment process is consistent with the results of Black (2002) and Ambaum and Hoskins (2002). Ambaum and Hoskins suggested that a positive NAO phase typically occurs as a result of a strengthened polar vortex associated with equatorward propagation of wave activity in the stratosphere. However, the present study reveals that a positive NAO pattern is generated as a result of wave- 1 reflection in the stratosphere, the subsequent growth of a high-latitude wave-1 pattern in the troposphere, and a midlatitude anomaly that arises from equatorward propagation. The westward phase progression of the high-latitude wave-1 pattern during the events suggests that the stratosphere can impact tropospheric weather through nonstationary planetaryscale waves. The role of quasi-stationary waves in forcing zonal wind anomalies in the troposphere during northern winter is consistent with DeWeaver and Nigam (2000) and the idealized simulations of Song and Robinson (2004). However, a detailed understanding of how wave reflection events impact the tropospheric jet requires further research.

Winter seasons with multiple downward wave coupling events, as indicated by a large cumulative negative wave coupling index, exhibit pronounced impacts on tropospheric climate that are consistent with those seen during the composite life cycle. In particular, the overall geopotential height, near-surface temperature, and mean sea level pressure anomalies are characteristic of the positive phase of the NAO. During years with large upward wave coupling, as indicated by a large cumulative positive index, the impacts are consistent with the negative phase of the NAO; however, the magnitude of the anomalies is much weaker than during years with a large downward wave coupling. This result suggests that upward wave propagation is not a process that directly leads to large impacts on the tropospheric circulation.

The seasonal impacts of large wave- 1 heat flux events were compared to JFM seasons with a weak and strong vortex as measured by the average zonal index in the stratosphere. The present analysis confirms previous 
studies (e.g., Baldwin and Dunkerton 2001; Polvani and Waugh 2004) that seasons with an extreme negative (positive) zonal index, and hence a weak (strong) stratospheric polar vortex, exhibit tropospheric anomalies consistent with the negative (positive) phase of the NAO with the magnitude of tropospheric anomalies being larger for weak vortex events. However, the present analysis also reveals that the tropospheric anomalies during years with an extreme positive zonal index are considerably weaker than during years with extreme negative wave coupling index, suggesting that wave reflection has a larger impact on the tropospheric circulation than the poleward refraction of planetary waves in the stratosphere. While the results suggest important links between the stratospheric seasonal indices and the phase of the NAO, further analysis with longer datasets is required to establish the statistical significance of the seasonal impacts.

The close link between the stratosphere and the NAO phase has been documented by many previous authors (e.g., Perlwitz and Graf 1995; Thompson and Wallace 1998; Kuroda and Kodera 1999). This link is conventionally associated with different phases of a single index, for example, weak and strong vortex events (Baldwin and Dunkerton 1999, 2001). The current results suggest that the connection between the stratosphere and the $\mathrm{NAO}$, including the impacts on tropospheric weather and climate, involves two distinct dynamical mechanisms that are best described by the negative tails of the wave coupling and zonal indices: stratospheric wave reflection and sudden stratospheric warmings due to wave absorption, respectively. The positive tails of the two indices have a much weaker impact on the tropospheresuggesting that the relationship between these two indices is not linear; namely, strong (weak) vortex events are not equivalent to downward (upward) wave coupling events. A detailed understanding of the relationship between the wave coupling and zonal indices during the different events is the subject of future investigation.

The impact of downward wave coupling events on tropospheric weather and climate has not been previously recognized because long time scale and latitudinal averages are typically applied when calculating the vortex events and their relation to heat flux events (Newman et al. 2001; Polvani and Waugh 2004; Limpasuvan et al. 2004). Our life cycle analysis shows that the impacts in the troposphere occur on weekly time scales consistent with wave propagation and predominately for wave 1 . In addition, the PC index times series encodes the latitudinal structure of the leading mode of heat flux variability and thus does not require any latitudinal averaging that may mask a large positive/negative meridional dipole pattern. Hurwitz et al. (2011) suggested that the recent strong (and cold) vortex winter (JFM) seasons in 1997 and 2011 are due to weak upward heat flux from the troposphere. An investigation of the role of wave reflection during these winters may provide additional insight. Several recent studies have discussed the role of wave interference in stratosphere-troposphere coupling (Garfinkel et al. 2011; Smith et al. 2010; Fletcher and Kushner 2011; Smith et al. 2011; Smith and Kushner 2012). Although linear interference is likely an important mechanism in the upper stratosphere where the wave- 1 heat flux variability occurs in the vicinity of the climatology, in the lower stratosphere and troposphere the impacts of wave reflection are focused in high latitudes where the climatological pattern is weak.

Overall, the results suggest that stratospheretroposphere coupling events should be defined using both wave coupling and zonal-mean indices and the impacts in the troposphere should be considered on weather and climate time scales (weekly to interannual). The implications of the connection between downward wave coupling and the NAO phase suggests that general circulation models that do not include a proper representation of stratosphere-troposphere dynamical coupling associated with wave reflection (e.g., Shaw and Perlwitz 2010), may be missing an amplifying factor of the NAO evolution, which is important for capturing trends in the NAO phase (e.g., Scaife et al. 2005).

Acknowledgments. TAS is supported by the National Science Foundation under Grant AGS-1129519. JP acknowledges support by NOAA's Climate Program Office. We thank the ECMWF for providing the ERAInterim reanalysis dataset, Dr. Nili Harnik for the use of her quasigeostrophic model and for helpful comments, Dr. Mark Baldwin for his EOF code, and finally to Drs. Limpasuvan and McDaniel for their help with the statistical significance calculations. The authors are grateful to three anonymous reviewers and Dr. Dave Thompson whose comments helped improve the manuscript.

\section{REFERENCES}

Ambaum, M. H. P., and B. J. Hoskins, 2002: The NAO tropospherestratosphere connection. J. Climate, 15, 1969-1978.

Baldwin, M. P., and T. J. Dunkerton, 1999: Propagation of the Arctic oscillation from the stratosphere to the troposphere. J. Geophys. Res., 104 (D24), 30 937-30 946.

— weather regimes. Science, 294, 581-584.

—, D. B. Stevenson, D. W. J. Thompson, T. J. Dunkerton, A. J. Charlton, and A. O'Neill, 2003: Stratospheric memory and skill of extended-range weather forecasts. Science, 301, 636-640.

- D. B. Stephenson, and I. T. Jolliffe, 2009: Spatial weighting and iterative projection methods for EOFs. J. Climate, 22, 234-243.

Black, R. X., 2002: Stratospheric forcing of surface climate in the Arctic Oscillation. J. Climate, 15, 268-277. 
Christiansen, B., 2001: Downward propagation of zonal mean zonal wind anomalies from the stratosphere to the troposphere: Model and reanalysis. J. Geophys. Res., 106 (D21), 27 307-27 322.

Dee, D. P., and Coauthors, 2011: The ERA-Interim reanalysis: Configuration and performance of the data assimilation system. Quart. J. Roy. Meteor. Soc., 137, 553-597.

DeWeaver, E., and S. Nigam, 2000: Do stationary waves drive the zonal-mean jet anomalies of the northern winter? J. Climate, 13, 2160-2176.

Fletcher, C. G., and P. J. Kushner, 2011: The role of linear interference in the annular mode response to tropical SST forcing. J. Climate, 24, 778-794.

Garfinkel, C. I., D. L. Hartmann, and F. Sassi, 2011: Tropospheric precursors of anomalous Northern Hemisphere stratospheric polar vortices. J. Climate, 23, 3282-3299.

Gerber, E. P., and Coauthors, 2012: Assessing and understanding the impact of stratospheric dynamics and variability on the earth system. Bull. Amer. Meteor. Soc., 93, 845-859.

Harnik, N., 2009: Observed stratospheric downward reflection and its relation to upward pulses of wave activity. J. Geophys. Res., 114, D08120, doi:10.1029/2008JD010493.

_ , and R. S. Lindzen, 2001: The effect of reflecting surfaces on the vertical structure and variability of stratospheric planetary waves. J. Atmos. Sci., 58, 2872-2894.

Hartley, D. E., J. T. Vilarin, R. X. Black, and C. A. Davis, 1998: A new perspective on the dynamical link between the stratosphere and troposphere. Nature, 391, 471-474.

Hartmann, D. L., J. M. Wallace, V. Limpasuvan, D. W. J. Thompson, and J. R. Holton, 2000: Can ozone depletion and global warming interact to produce rapid climate change? Proc. Natl. Acad. Sci. USA, 97, 1412-1417.

Haynes, P. H., 2005: Stratospheric dynamics. Annu. Rev. Fluid Mech., 37, 263-293.

Hurrell, J. W., Y. Kushnir, M. Visbeck, and G. Ottersen, 2003: An overview of the North Atlantic Oscillation. The North Atlantic Oscillation: Climate Significance and Environmental Impact, Geophys. Monogr., Vol. 134, Amer. Geophys. Union, 1-35.

Hurwitz, M. M., P. A. Newman, and C. I. Garfinkel, 2011: The Arctic vortex in March 2011: A dynamical perspective. Atmos. Chem. Phys., 11, 11 447-11 453.

Kuroda, Y., and K. Kodera, 1999: Role of planetary waves in the stratosphere-troposphere coupled variability in the northern hemisphere winter. Geophys. Res. Lett., 26, 2375-2378.

Limpasuvan, V., D. J. Thompson, and D. L. Hartmann, 2004: The life cycle of the Northern Hemisphere sudden stratospheric warmings. J. Climate, 17, 2584-2596.

Newman, P. A., E. R. Nash, and J. E. Rosenfield, 2001: What controls the temperature in the Arctic stratosphere in the spring? J. Geophys. Res., 106 (D17), 19 999-20 010.

North, G. R., T. L. Bell, R. F. Cahalan, and F. J. Moeng, 1982: Sampling errors in the estimation of empirical orthogonal functions. Mon. Wea. Rev., 110, 699-706.
Perlwitz, J., and H.-F. Graf, 1995: The statistical connection between tropospheric and stratospheric circulation of the Northern Hemisphere in winter. J. Climate, 8, 2281-2295.

- , and N. Harnik, 2003: Observational evidence of a stratospheric influence on the troposphere by planetary wave reflection. J. Climate, 16, 3011-3026.

- , and - 2004: Downward coupling between the stratosphere and troposphere: The relative roles of wave and zonal mean processes. J. Climate, 17, 4902-4909.

Plumb, R. A., and K. Semeniuk, 2003: Downward migration of extratropical zonal wind anomalies. J. Geophys. Res., 108, 4223, doi:10.1029/2002JD002773.

Polvani, L. M., and P. J. Kushner, 2002: Tropospheric response to stratospheric perturbations in a relatively simple general circulation model. Geophys. Res. Lett., 29, 1114, doi:10.1029/ 2001GL014284.

- and D. Waugh, 2004: Upward wave activity flux as a precursor to extreme stratospheric events and subsequent anomalous surface weather regimes. J. Climate, 17, 35483554 .

Randel, W. J., 1987: A study of planetary waves in the southern winter troposphere and stratosphere. Part I: Wave structure and vertical propagation. J. Atmos. Sci., 44, 917-935.

Scaife, A. A., J. R. Knight, G. K. Vallis, and C. K. Folland, 2005: A stratospheric influence on the winter NAO and North Atlantic surface climate. Geophys. Res. Lett., 32, L18715, doi:10.1029/ 2005GL023226.

Shaw, T. A., and T. G. Shepherd, 2008: Raising the roof. Nat. Geosci., 1, 12-13.

, and J. Perlwitz, 2010: The impact of stratospheric model configuration on planetary-scale waves in Northern Hemisphere winter. J. Climate, 23, 3369-3389.

$[$, -, and N. Harnik, 2010: Downward wave coupling between the stratosphere and troposphere: The importance of meridional wave guiding and comparison with zonal-mean coupling. J. Climate, 23, 6365-6381.

Smith, K. L., and P. J. Kushner, 2012: Linear interference and the initiation of extratropical stratosphere-troposphere interactions. J. Geophys. Res., 117, D13107, doi:10.1029/2012JD017587.

, C. G. Fletcher, and P. J. Kushner, 2010: The role of linear interference in the annular mode response to extratropical surface forcings. J. Climate, 23, 6036-6050.

, P. J. Kushner, and J. Cohen, 2011: The role of linear interference in northern annular mode variability associated with Eurasian snow cover extent. J. Climate, 24, 61856202

Song, W., and W. A. Robinson, 2004: Dynamical mechanisms for stratospheric influences on the troposphere. J. Atmos. Sci., 61, 1711-1725.

Thompson, D. W. J., and J. M. Wallace, 1998: The Arctic Oscillation signature in the wintertime geopotential height and temperature fields. Geophys. Res. Lett., 25, 1297-1300. 